\title{
LATTÈS-TYPE MAPPINGS ON COMPACT MANIFOLDS
}

\author{
LAURA ASTOLA, RIIKKA KANGASLAMPI, AND KIRSI PELTONEN
}

\begin{abstract}
A uniformly quasiregular mapping acting on a compact Riemannian manifold distorts the metric by a bounded amount, independently of the number of iterates. Such maps are rational with respect to some measurable conformal structure and there is a Fatou-Julia type theory associated with the dynamical system obtained by iterating these mappings. We study a rich subclass of uniformly quasiregular mappings that can be produced using an analogy of classical Lattès' construction of chaotic rational functions acting on the extended plane $\overline{\mathbb{C}}$. We show that there is a plenitude of compact manifolds that support these mappings. Moreover, we find that in some cases there are alternative ways to construct this type of mapping with different Julia sets.
\end{abstract}

\section{INTRODUCTION}

In recent years a theory analogous to that of the iteration of rational functions in the Riemann sphere $\overline{\mathbb{C}}$, distinct from that of iteration of holomorphic functions in several complex variables has been developed in higher dimensions. A uniformly quasiregular mapping (uqr mapping) is a mapping $f: M \rightarrow M$ acting on a compact Riemannian manifold $M$ in such a way that all the iterates are quasiregular with a uniform bound on the distortion constant. The basic theory of quasiregular mappings $f: D \rightarrow \overline{\mathbb{R}}^{n}$ defined in Euclidean domains $D \subset \overline{\mathbb{R}}^{n}$ is extensively studied in [13. The surprising fact that there indeed exist non-injective examples of uqr maps acting on sphere $\overline{\mathbb{R}}^{n}$ was first discovered by Iwaniec and Martin [4]. Moreover, it was later discovered [12] that there are many other compact manifolds than just the ordinary sphere that support this type of mapping. Recently these mappings have been constructed even in the presence of a subriemannian metric in case of the Heisenberg group [1. All these examples are variations of the so called trapping method originally invented in [4] and further crystallized in [9]. There the idea is to build a conformal trap, an area, where all the points whose neighbourhood is distorted, land after finite, uniformly bounded, number of iterates. Once hitting the trap, the points will also stay conformally inside the trap under further iterates. This method always produces mappings, whose Julia set is a Cantor set. If a uqr mapping $f$ exists on a compact Riemannian manifold, it is always possible to construct a bounded measurable conformal structure that remains invariant under the action of the semigroup $\Lambda$ of iterates $f^{n}, n \in \mathbb{N}$.

Received by the editors August 23, 2010.

2010 Mathematics Subject Classification. Primary 53A30, 53C20; Secondary 30C65.

Key words and phrases. Uniformly quasiregular mapping, Lattès-type mapping, Julia set, conformal structure. 
In case of the Heisenberg group equipped with the natural subriemannian CarnotCarathéodory metric this corresponds to the existence of a horizontal invariant conformal structure and equivalently existence of a non-standard equivariant measurable CR structure [1].

In addition to the above trapping method, another way to produce uqr maps is also known. In 1997 and 1998, Mayer discovered an important family of examples of uniformly quasiregular mappings; see [10] and 11. They are analogues of the rational functions that are called critically finite with parabolic orbifold. Mayer generalized Lattès' construction of so-called chaotic rational maps [8]. Moreover, he showed that in addition to the chaotic analogue of the Lattès mapping on the $n$-sphere $\overline{\mathbb{R}}^{n}$ there exist analogues of planar power mappings $z \mapsto z^{d}$ as well as Tchebychev polynomials like $z \mapsto z^{2}-2$ showing that, with this respect, the theory of uqr maps in higher dimensional spheres is as equally rich as in two dimensions. The uniformly quasiregular counterparts for power mappings have a codimension 1 sphere as a Julia set with origin and infinity as super-attracting and completely invariant fixed points. The uqr counterpart of Tchebychev polynomials have a codimension 1 closed unit disk as a Julia set and completely invariant fixed point infinity. In what follows, we recall these basic constructions and develop them for further examples.

It is interesting to note, that the only manifolds that we know to support Lattèstype maps as well as uqr maps that arise through the trapping method, are the ordinary sphere and the projective space in odd dimensions. Since all spherical space forms support trapping method [12, it remains a challenging open question whether among these spaces Lattès-type mappings could be produced. On the other hand, we do not know whether we have reached all the compact manifolds outside the spherical space forms that support Lattès-type maps either. Towards classification of manifolds supporting Lattès-type uqr maps, there is an interesting characterization by Mayer [11] in terms of invariant conformal structures. Although he formulated the theorem only for the sphere, the statement holds also for compact Riemannian manifolds.

The existence of a non-injective uqr map is a global obstruction for the manifold. It is known (see [5] and [7]) that if such a map exists, then the manifold $M^{n}$ has to be elliptic; that is, there must exist a non-constant quasiregular mapping $f$ : $\mathbb{R}^{n} \rightarrow M^{n}$. In dimension three this is a characterization [7. In higher dimensions the situation is open. We do not know, for example, whether an elliptic manifold $S^{2} \times S^{2} \# S^{2} \times S^{2}[14$ supports non-injective uqr maps.

The paper is organized as follows: In section 2 we fix the notation and recall the basic definitions and previous results needed here. In section 3 we study the spaces that come up in dimension 3. In further sections 4 and 5, the dimensions 4 and 5 are treated separately to focus on increasing flexibility of mappings and the variety of possible Julia sets. In the last chapter we outline the constructions in higher dimensions.

\section{Notation AND PRELIMINARIES}

Let $D \subset \overline{\mathbb{R}}^{n}$ be a domain and $f: D \rightarrow \overline{\mathbb{R}}^{n}$ a non-constant mapping of the Sobolev class $W_{l o c}^{1, n}(D)$. We consider only orientation-preserving mappings, which 
means that the Jacobian determinant $J_{f}(x) \geq 0$ for a.e. $x \in D$. Such a mapping is said to be $K$-quasiregular, where $1 \leq K<\infty$, if

$$
\max _{|h|=1}\left|f^{\prime}(x) h\right| \leq K \min _{|h|=1}\left|f^{\prime}(x) h\right|
$$

for a.e. $x \in D$, when $f^{\prime}$ is the formal matrix of weak derivatives.

We can generalize this definition to Riemannian manifolds with the help of bilipschitz-continuous coordinate charts. Let $M$ and $N$ be smooth $n$-dimensional Riemannian manifolds. A non-constant mapping $f: M \rightarrow N$ is $K$-quasiregular if for every $\varepsilon>0$ and every $m \in M$ there exists bilipschitz-continuous charts $(U, \varphi)$, $m \in U$, and $(V, \psi), f(m) \in V$, so that the mapping $\psi \circ f \circ \varphi^{-1}$ is $(K+\varepsilon)$-quasiregular.

A non-constant quasiregular mapping can be redefined on a set of measure zero so as to be continuous, open and discrete 13 . We will henceforth assume that quasiregular mappings always have these properties. The branch set $B_{f}$ of the mapping $f: M \rightarrow N$ is the set of those points $x \in M$ where $f$ is not locally homeomorphic.

Let $M$ be a compact Riemannian $n$-manifold. A non-injective mapping $f$ from a domain $D \subset M$ onto itself is called uniformly quasiregular (uqr) if there exists a constant $1 \leq K \leq \infty$ such that all the iterates $f^{k}$ are $K$-quasiregular.

A $K$-quasiregular semigroup is a family of $K$-quasiregular mappings, which is closed under composition of the mappings. We define Julia and Fatou sets of such semigroups as follows:

Definition 2.1. Let $\Lambda$ be a quasiregular semigroup. Then the Fatou set of $\Lambda$ is

$$
\begin{array}{ll}
\mathcal{F}(\Lambda)=\{x \in M: & \text { there exists an open set } U \subset M \\
& \text { such that } x \in U \text { and } \Lambda \mid U \text { is normal }\} .
\end{array}
$$

The Julia set of the family $\Lambda$ is $\mathcal{J}(\Lambda)=M \backslash \mathcal{F}(\Lambda)$.

By the definition, Fatou sets are open, and therefore Julia sets are closed. If the family $\Lambda$ consists of iterates of a mapping $f$, that is $\Lambda=\left\{f^{k} \mid k=1,2, \ldots\right\}$, we call these sets simply Fatou and Julia sets of the mapping $f$, denoted by $\mathcal{F}_{f}$ and $\mathcal{J}_{f}$. Fatou and Julia sets are completely invariant [7]. We call a uqr mapping chaotic if the Julia set is the whole manifold $M$.

Since the derivative of a uqr mapping need not exist at a given point, the classification of different fixed point types is not available as for holomorphic maps. To see how this difficulty can be overcome by establishing Lipschitz estimates near fixed points, see 3 .

Let $G$ be a measurable conformal structure on $M$. By that we mean a measurable and bounded map $G: M \rightarrow S(n)$, where $S(n)$ is the space of symmetric $n \times n$ positive definite matrices of determinant 1 . Moreover, for almost every point $x \in M$, $G(x)$ is a linear automorphism

$$
G(x): T_{x} M \rightarrow T_{x} M
$$

of the inner product space $T_{x} M$ given by a fixed Riemannian metric $\langle$,$\rangle satisfying$ a uniform ellipticity condition

$$
K^{-1}|\xi|^{2} \leq\langle G(x) \xi, \xi\rangle \leq K|\xi|^{2}
$$


with $K \geq 1$ independent of $x$. Such a conformal structure is a Riemannian metric with measurable coefficients. The normalization requirement $\operatorname{det} G(x)=1$ a.e. is due to the fact that from the point of view of quasiconformal maps, two structures, $G$ and $G^{\prime}$ are equivalent if they differ a.e. by a constant: $G(x)=\lambda_{x} G^{\prime}(x)$ a.e. in $M$.

For $G$ satisfying (2.1), the solutions of the equation

$$
D^{t} f(x) G(f(x)) D f(x)=J_{f}(x)^{\frac{2}{n}} G(x)
$$

for mappings of Sobolev class $W_{l o c}^{1, n}(M)$ form a semigroup under composition. Each such solution is a $K^{2}$-quasiregular mapping of $M$, where $K$ is determined by the ellipticity bound (2.1) on $G$. We call the semigroup of non-constant solutions to (2.2) the $G$-rational mappings or simply rational mappings if $G$ is understood. Conformal structure $G$ is referred to as an invariant conformal structure for this semigroup. In dimension 2 and $G=I$ the identity matrix, the differential equation (2.2) reduces to the usual Cauchy-Riemann equations and such solutions necessarily represent rational endomorphisms of $\overline{\mathbb{C}}$. Conversely, if $f$ is a uqr mapping, then there exists an equivariant conformal structure for the semigroup $\Lambda=\left\{f^{n} \mid n \in \mathbb{N}\right\}$ generated by $f$. The proof is analogous to the construction given for two dimensional quasiconformal groups in [16] and is presented in [4]. For further discussion on conformal structures, see [5].

We recall that, in the plane, a Lattès map is obtained by semi-conjugating an expanding similarity with an elliptic function. The original example given by Lattès [8] was the rational map

$$
z \mapsto \frac{\left(z^{2}+1\right)^{2}}{4 z\left(z^{2}-1\right)} .
$$

To end up with this and a whole family of similar chaotic examples in the extended complex plane, see for example [2], where the properties of the two periodic Weierstrass $\wp$-function are used to characterize this class. The geometric nature behind the algebraic properties can be captured for uqr mappings of $\overline{\mathbb{R}}^{n}$ and more general compact manifolds $M$, the principal tool being automorphic functions:

Definition 2.2. Let $\Gamma$ be a discrete group of isometries of $\mathbb{R}^{n}$. A mapping $h$ : $\mathbb{R}^{n} \rightarrow M$ is automorphic with respect to $\Gamma$ in the strong sense if

(1) $h \circ \gamma=h$ for any $\gamma \in \Gamma$,

(2) $\Gamma$ acts transitively on the fibres $\mathcal{O}_{y}=h^{-1}(y)$.

By the latter condition we mean that for any two points $x_{1}, x_{2}$ with $h\left(x_{1}\right)=$ $h\left(x_{2}\right)$ there is an isometry $\gamma \in \Gamma$ such that $x_{2}=\gamma\left(x_{1}\right)$. We have the following theorem by Iwaniec and Martin [5, pp. 501-502]. The proof in [5] is written for the case $M=\overline{\mathbb{R}}^{n}$, but it holds more generally on a Riemannian manifold $M$ without changes.

Theorem 2.3. Let $\Gamma$ be a discrete group such that $h: \mathbb{R}^{n} \rightarrow M$ is automorphic with respect to $\Gamma$ in the strong sense. If there is a similarity $A=\lambda \mathcal{O}, \lambda \in \mathbb{R}, \lambda \neq 0$, and $\mathcal{O}$ an orthogonal transformation, such that

$$
A \Gamma A^{-1} \subset \Gamma,
$$

then there is a unique solution $f: h\left(\mathbb{R}^{n}\right) \rightarrow h\left(\mathbb{R}^{n}\right)$ to the Schröder functional equation

$$
f \circ h=h \circ A \text {. }
$$

If $h$ is quasiregular, the mapping $f$ is uniformly quasiregular. 
By the condition $A \Gamma A^{-1} \subset \Gamma$ it is simply meant that for any $\gamma \in \Gamma$ there exists a $\gamma^{\prime} \in \Gamma$ such that $A \gamma(x)=\gamma^{\prime} A(x)$ for any $x$. The idea of the proof presented in [5] is that if $h$ is automorphic with respect to a discrete group, it does not recognize whether the space has been "moved" or not. If, in addition, $A$ does not disturb the action of the group, there is a solution to the Schröder equation. Note that following from (2.3) we have the equation $f^{k} \circ h=h \circ A^{k}$ for all $k$. Thus the dilatation of the uqr mapping $f^{k}$ is exactly the dilatation of $h^{2}$ for all $k$ :

$$
K(f)=K\left(f^{k}\right)=K\left(h^{2}\right) .
$$

Geometrically the Weierstrass $\wp$-function is obtained by first mapping the square $Q=] 0,1\left[^{2}\right.$ quasiconformally onto the northern hemisphere of $\mathbb{S}^{2}$ and then extending this mapping to the complex plane using repeated reflections on the sides of $Q$ and on the equator in the range. The group $\Gamma$ is given by the isometries that come from an even number of reflections at the sides of $Q$. By multiplying with $A: A(z)=2 z$ the resulting uqr mapping $f_{\text {Lat }}$ defined as a solution of (2.3) is chaotic.

Three different Lattès-type mappings exist in the holomorphic case in the extended complex plane. In addition to the above mentioned chaotic rational functions, the original example $f_{\text {Lat }}$ of Lattès, the power mappings $z \mapsto z^{d}$ and Tchebychev polynomials like $z \mapsto z^{2}-2$ belong to this class. The Julia set of the power mapping is the unit circle, and the Fatou set has two components, while the Tchebychev polynomials have closed a line segment as the Julia set and the Fatou set has one component only. All these three types have analogues on higher dimensional spheres.

We formulate the following characterization of Lattès-type mappings:

Theorem 2.4 ([11]). A non-injective uniformly quasiregular mapping $f$ of $M^{n}$, $n \geq 3$, is of Lattès type if and only if it has an $f$-invariant conformal structure which is flat at a repelling fixed point of some iterate $f^{k}$ of the mapping.

We recall that the flatness of a conformal structure $G$ at a point $p$ means that there exists a neighbourhood $U$ of $p$ and a quasiconformal mapping $\varphi$ defined on $U$ such that

$$
D^{t} \varphi(x) G(f(x)) D \varphi(x)=J_{\varphi}(x)^{\frac{2}{n}} I(x)
$$

holds for a.e. $x \in U$. The symbol $I$ denotes the $n \times n$ identity matrix. Although the above theorem is formulated for the sphere only in [1] the proof generalizes to compact manifolds verbatim.

In many examples below we end up with chaotic Lattès-type mappings. To this direction, it is interesting to note that the situation is very rigid with respect to possible conformal structures.

Theorem 2.5 ([10]). Let $f$ be a chaotic Lattès-type mapping acting on manifold $M^{n}$ and satisfying the Schröder functional equation (2.3). Then a conformal structure $G$ is $f$-invariant if and only if the pull back of $G$ under $h$,

$$
H(x)=J_{h}(x)^{-\frac{2}{n}} D^{t} h(x) G(h(x)) D h(x),
$$

a.e., in $\mathbb{R}^{n}$ is a constant $\Gamma$ - and A-invariant structure. Moreover, when $n \geq 3$, then $H$ has a diagonal form

$$
H=\operatorname{diag}\left(m_{1}, m_{2}, \ldots, m_{n}\right), \quad m_{i}>0 \text { and } m_{1} m_{2} \cdots m_{n}=1 .
$$


The proof for this general setting follows as the spherical case presented in [10] verbatim.

\section{Constructions in three Dimensions}

In dimension three it is possible to construct uqr mappings with Julia sets that have the same characteristics as the Julia sets of uqr maps in the plane; that is, chaotic mappings and mappings with lower dimensional manifolds or Cantor sets as Julia sets. Lattès-type uqr mappings can have Julia sets of the first two types, Cantor sets can be obtained e.g. with the conformal trap method. Next we construct some examples in detail.

3.1. Sphere $\mathbb{S}^{3}$. There is a number of examples of Lattès-type uqr mappings on the three dimensional sphere by Mayer (see e.g. [10]). He constructs chaotic uqr mappings acting on $\mathbb{S}^{3}$ and analogues of power mappings with Julia set $\mathbb{S}^{2}$. Some of the constructions are presented generally on $\mathbb{S}^{n}$. We recall three mappings on $\mathbb{S}^{3}$ that are essential in later examples. The first one is a generalization of the power mapping acting in the plane. It is induced by the Zorich mapping, and has the Julia set $\mathbb{S}^{2}$. The second one is chaotic, and it is based on the cubical decomposition of the space. The last one uses an analogue of the Tchebychev polynomial, and has a closed 2-disk $\overline{\mathbb{D}} \times\{0\}$ as the Julia set.

3.1.1. Power mapping. Let us first show how a 4-to-1 uqr mapping arises on $\mathbb{S}^{3}$ via the Zorich mapping, the higher dimensional counterpart of the planar exponential function, as presented by Rickman [13, p. 15]) and later Mayer [10. Denote the Zorich mapping by $h_{Z}: \mathbb{R}^{3} \rightarrow \mathbb{R}^{3} \backslash\{0\}$. This mapping is constructed by first subdividing space into infinite cylinders with integer lattice base in the $x_{1} x_{2}$-plane. Take a slice $Z=\left\{\left(x_{1}, x_{2}, x_{3}\right) \mid 0<x_{1}, x_{2} \leq 1, x_{3} \in \mathbb{R}\right\}$ in $\mathbb{R}^{3}$ first to an infinite cylinder of radius 1 by a bilipschitz radial stretching, and then map the round cylinder quasiconformally onto the upper half-space $\mathbb{H}$ by the mapping $\left(r, \varphi, x_{3}\right) \mapsto$ $\left(\exp \left(x_{3}\right), \varphi, \frac{\pi r}{2}\right)$, where cylindrical and spherical coordinates, respectively, are used. The mapping $h_{Z}$ is obtained by extending this map to a quasiregular map of $\mathbb{R}^{3}$ to $\mathbb{R}^{3} \backslash\{0\}$ by using reflections on faces of $Z$ and $\partial \mathbb{H}$. So $h_{Z}$ alternately maps neighbouring cylinders to the upper and lower half-space. The branch set of $h_{Z}$ consists of the edges of $Z$ and the reflected cylinders, i.e. $B_{h}=\mathbb{Z}^{2} \times \mathbb{R}$.

If we semiconjugate the mapping $A_{2}: x \mapsto 2 x$ in $\mathbb{R}^{3}$ with the Zorich mapping $h_{Z}$, a 4-to-1 mapping $f_{\text {pwr }}: \mathbb{R}^{3} \backslash\{0\} \rightarrow \mathbb{R}^{3} \backslash\{0\}$ is induced via equation (2.3).

The mapping is further extended continuously to the origin and the infinity by defining $f_{p w r}(0)=0$ and $f_{p w r}(\infty)=\infty$. Thus $f_{p w r}$ is a mapping acting on the 3 -sphere, $f_{p w r}: \mathbb{S}^{3} \rightarrow \mathbb{S}^{3}$. By Theorem 2.3 this mapping is uniformly quasiregular. It is a generalization of the power mapping in the plane (see Figure 1).

The Julia set is the image of the $x_{1} x_{2}$-plane in $\mathbb{R}^{3}$ under the Zorich mapping, that is, a 2 -sphere of radius 1 , centered at the origin. The point $h_{Z}(0)$ is a repelling fixed point of $f_{p w r}$. The mapping $f_{p w r}$ has two basins of attraction, the inside and the outside of the Julia set, the origin and infinity being superattractive fixed points. The branch set consists of six half-lines that meet at the origin and infinity. They correspond to images of six vertical lines in $\mathbb{R}^{3}$ under the Zorich mapping: four lines in the middle of the faces of $Z$, one through its center and one through the center of its neighbouring cylinder. The local index is 2 along the branch lines outside the origin and infinity and 4 at the origin and infinity. 

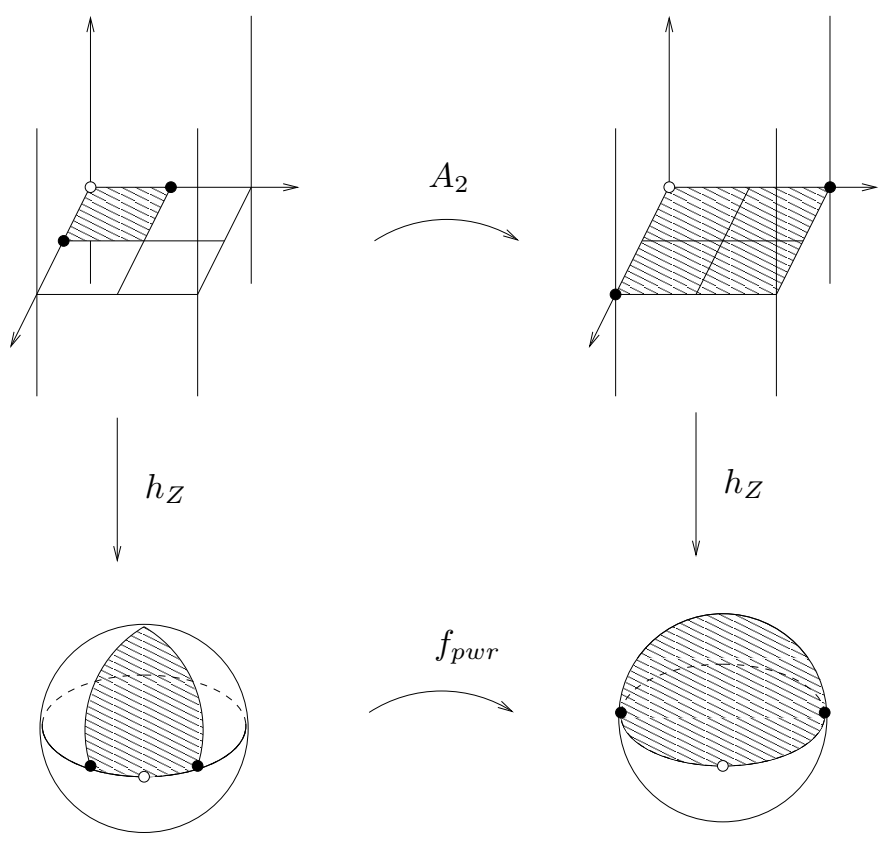

FiguRE 1. Construction of a uqr mapping $f_{p w r}$ on $\mathbb{S}^{3}$

If we semiconjugate above a general dilation $A_{\lambda}: x \mapsto \lambda x$ in $\mathbb{R}^{3}, \lambda=2,3, \ldots$ with the Zorich mapping $h_{Z}$, a $\lambda^{2}$-to-1 mapping $f_{\text {pwr }}^{\lambda}: \mathbb{R}^{3} \backslash\{0\} \rightarrow \mathbb{R}^{3} \backslash\{0\}$ is induced via equation (2.3). Note that while the branch set $B_{h}$ of the Zorich mapping constitutes the edges of the cylindrical decomposition of the space, the branch set of the induced power mapping consists of $2\left(\lambda^{2}-1\right)$ half lines meeting at the origin and infinity. More precisely, the branch set of the power mapping is the set $h\left(\left(A_{\lambda}^{-1} B_{h}\right) \backslash B_{h}\right)$. The local index is 2 along the branch lines outside the origin and infinity and $\lambda^{2}$ at the origin and infinity.

3.1.2. Chaotic mapping. The classical example $f_{\text {Lat }}$ in the complex plane can be easily adapted to higher dimensions. We replace $Q=[0,1)^{2}$ by the cube $Q=$ $[0,1)^{3}$ and find a quasiconformal homeomorphism $h$ from this cube to the upper hemisphere of $\mathbb{S}^{3}$. Then we again extend this map $h$ using reflections with respect to the faces of $Q$ and inversion on the equator of $\mathbb{S}^{3}$ in the range. The function $h: \mathbb{R}^{3} \rightarrow \mathbb{S}^{3}$ that we obtain, is a quasimeromorphic version of the Weierstrass $\wp$ function. This map is automorphic with respect to a crystallographic group $\Gamma \subset \operatorname{Isom}\left(\mathbb{R}^{3}\right)$. The branch set $B_{h}$ of $h$ is the one skeleton of the cubical subdivision of space.

To describe the identifications in the above subdivision of $\mathbb{R}^{3}$ we first find that the even integer lattice gives cubes congruent to $[0,2)^{3}$ under translations $\left(x_{1}, x_{2}, x_{3}\right) \mapsto$ $\left(x_{1}+2, x_{2}, x_{3}\right),\left(x_{1}, x_{2}, x_{3}\right) \mapsto\left(x_{1}, x_{2}+2, x_{3}\right)$ and $\left(x_{1}, x_{2}, x_{3}\right) \mapsto\left(x_{1}, x_{2}, x_{3}+2\right)$. Each such cube is further divided into eight unit cubes. Let $e_{1}, e_{2}$ and $e_{3}$ denote the three orthogonal unit vectors in $\mathbb{R}^{3}$, and let $x_{1}, x_{2}, x_{3} \in \mathbb{R}$ be coordinates of 
an arbitrary point in $\mathbb{R}^{3}$. All the points of the set

$$
\begin{aligned}
& \left\{\left(x_{1}+2 n_{1}\right) e_{1}+\left(x_{2}+2 n_{2}\right) e_{2}+\left(x_{3}+2 n_{3}\right) e_{3},\right. \\
& \quad\left(2-x_{1}+2 m_{1}\right) e_{1}+\left(2-x_{2}+2 m_{2}\right) e_{2}+\left(x_{3}+2 m_{3}\right) e_{3}, \\
& \left(x_{1}+2 k_{1}\right) e_{1}+\left(2-x_{2}+2 k_{2}\right) e_{2}+\left(2-x_{3}+2 k_{3}\right) e_{3}, \\
& \left(2-x_{1}+2 l_{1}\right) e_{1}+\left(x_{2}+2 l_{2}\right) e_{2}+\left(2-x_{3}+2 l_{3}\right) e_{3} \\
& \left.\quad \mid n_{i}, m_{i}, k_{i}, l_{i} \in \mathbb{Z}, i=1,2,3\right\}
\end{aligned}
$$

will be identified under two repeated reflections with respect to 2 -faces of the (unit) cubical subdivision to represent the same point of the manifold $\mathbb{S}^{3}$. This identification induces a branched covering $h: \mathbb{R}^{3} \rightarrow \mathbb{S}^{3}$. Each cube congruent to $[0,2)^{3}$ covers space $\mathbb{S}^{3}$ four times.

If the dilation $A_{2}$ is taken in equation (2.3), then an 8-to-1 mapping $f_{\text {cube }} \in$ $U Q R\left(\mathbb{S}^{3}\right)$ is obtained as a solution. It follows from the cocompactness of this group that the solutions $f_{\text {cube }}$ are chaotic: the Julia set is the whole sphere. Again the point $h(0)$ is a repelling fixed point of $f_{\text {cube }}$. The branch set of $f_{\text {cube }}$ is the set $\left.h\left(\left(A_{2}^{-1} B_{h}\right) \backslash B_{h}\right)\right)$.

If we semiconjugate above a general $A_{\lambda}: x \mapsto \lambda x$ in $\mathbb{R}^{3}, \lambda=2,3, \ldots$ with the mapping $h$, a $\lambda^{3}$-to-1 mapping $f_{\text {cube }}^{\lambda}: \mathbb{S}^{3} \rightarrow \mathbb{S}^{3}$ is induced via equation (2.3).

The fact that the mapping $f_{\text {cube }}^{\lambda}$ is well defined for the arbitrary integer $\lambda>1$ can be seen as follows. Let $x$ be a point on $\mathbb{S}^{3}$ and $x_{1} e_{1}+x_{2} e_{2}+x_{3} e_{3} \in \mathbb{R}^{3}$, where $x_{1}, x_{2}, x_{3} \in[0,2)$, be one of its preimages under $h$. We see that the whole preimage set of the point $x$ under the branched covering map $h$ is

$$
\begin{aligned}
h^{-1}(x)=\{ & \left(x_{1}+2 n\right) e_{1}+\left(x_{2}+2 m\right) e_{2}+\left(x_{3}+2 k\right) e_{3}, \\
& \left(2-x_{1}+2 n^{\prime}\right) e_{1}+\left(2-x_{2}+2 m^{\prime}\right) e_{2}+\left(x_{3}+2 k^{\prime}\right) e_{3}, \\
& \left(x_{1}+2 n^{\prime \prime}\right) e_{1}+\left(2-x_{2}+2 m^{\prime \prime}\right) e_{2}+\left(2-x_{3}+2 k^{\prime \prime}\right) e_{3}, \\
& \left(2-x_{1}+2 \tilde{n}\right) e_{1}+\left(x_{2}+2 \tilde{m}\right) e_{2}+\left(2-x_{3}+2 \tilde{k}\right) e_{3} \\
& \left.\mid n, m, k, n^{\prime}, m^{\prime}, k^{\prime}, n^{\prime \prime}, m^{\prime \prime}, k^{\prime \prime}, \tilde{n}, \tilde{m}, \tilde{k} \in \mathbb{Z}\right\} .
\end{aligned}
$$

Consequently, the set

$$
\begin{aligned}
A_{\lambda}\left(h^{-1}(x)\right)=\{ & \lambda\left(x_{1}+2 n\right) e_{1}+\lambda\left(x_{2}+2 m\right) e_{2}+\lambda\left(x_{3}+2 k\right) e_{3}, \\
& \lambda\left(2-x_{1}+2 n^{\prime}\right) e_{1}+\lambda\left(2-x_{2}+2 m^{\prime}\right) e_{2}+\lambda\left(x_{3}+2 k^{\prime}\right) e_{3}, \\
& \lambda\left(x_{1}+2 n^{\prime \prime}\right) e_{1}+\lambda\left(2-x_{2}+2 m^{\prime \prime}\right) e_{2}+\lambda\left(2-x_{3}+2 k^{\prime \prime}\right) e_{3}, \\
& \lambda\left(2-x_{1}+2 \tilde{n}\right) e_{1}+\lambda\left(x_{2}+2 \tilde{m}\right) e_{2}+\lambda\left(2-x_{3}+2 \tilde{k}\right) e_{3} \\
& \left.\mid n, m, k, n^{\prime}, m^{\prime}, k^{\prime}, n^{\prime \prime}, m^{\prime \prime}, k^{\prime \prime}, \tilde{n}, \tilde{m}, \tilde{k} \in \mathbb{Z}\right\} \\
\subset\{ & \left(\lambda x_{1}+2 n\right) e_{1}+\left(\lambda x_{2}+2 m\right) e_{2}+\left(\lambda x_{3}+2 k\right) e_{3}, \\
& \left(2-\lambda x_{1}+2 n^{\prime}\right) e_{1}+\left(2-\lambda x_{2}+2 m^{\prime}\right) e_{2}+\left(\lambda x_{3}+2 k^{\prime}\right) e_{3}, \\
& \left(\lambda x_{1}+2 n^{\prime \prime}\right) e_{1}+\left(2-\lambda x_{2}+2 m^{\prime \prime}\right) e_{2}+\left(2-\lambda x_{3}+2 k^{\prime \prime}\right) e_{3}, \\
& \left(2-\lambda x_{1}+2 \tilde{n}\right) e_{1}+\left(\lambda x_{2}+2 \tilde{m}\right) e_{2}+\left(2-\lambda x_{3}+2 \tilde{k}\right) e_{3} \\
& \left.\mid n, m, k, n^{\prime}, m^{\prime}, k^{\prime}, n^{\prime \prime}, m^{\prime \prime}, k^{\prime \prime}, \tilde{n}, \tilde{m}, \tilde{k} \in \mathbb{Z}\right\}
\end{aligned}
$$

becomes a single point $y:=y_{1} e_{1}+y_{2} e_{2}+y_{3} e_{3}$ in $\mathbb{S}^{3}$ under the mapping $h$, where $y_{i} \in[0,2), y_{i}=x_{i} \bmod 2, i=1,2,3,4$. Thus $A_{\lambda}$ descends to a well-defined mapping $f_{\text {cube }}^{\lambda}: \mathbb{S}^{3} \rightarrow \mathbb{S}^{3}$. 
A similar method works on higher dimensional spheres as well. One just takes an $n$-dimensional cube instead of the 3 -cube in the construction. The details for the identifications are shown in sections 4.1, 5 and 6 .

3.1.3. Tchebychev mapping. Yet another example on $\mathbb{S}^{3}$ that will be used in later constructions is based on the analogues of Tchebychev polynomials. The obtained uqr mapping has the Julia set $\overline{\mathbb{D}} \times\{0\}=\left\{\left(x_{1}, x_{2}, 0\right) \mid x_{1}^{2}+x_{2}^{2} \leq 1\right\} \subset \overline{\mathbb{R}}^{3}$. The following construction has been presented in [11].

Let $Z=] 0,1\left[^{2} \times \mathbb{R}\right.$ and denote the lower half by $Z^{-}=Z \cap\left\{x_{3}<0\right\}$. From the above construction of Zorich's mapping (Section 3.1.1) we have a quasiconformal mapping $h_{Z}$ from the cylinder $Z$ onto the upper half-space $\mathbb{H}=\left\{\left(x_{1}, x_{2}, x_{3}\right) \in\right.$ $\left.\mathbb{R}^{3} \mid x_{3}>0\right\}$, such that $h_{Z}\left(Z^{-}\right)=\mathbb{H} \cap \mathbb{B}(0,1)$. The half-ball is conformally equivalent to the quarter-space $\left\{\left(x_{1}, x_{2}, x_{3}\right) \in \mathbb{R}^{3} \mid x_{1}>0, x_{3}>0\right\}$ via inversion with respect to sphere $S((1,0,0), \sqrt{2})$ :

$$
I: x \mapsto \frac{2(x-q)}{|x-q|^{2}}+q, \quad q=(1,0,0),
$$

followed by a reflection $r:\left(x_{1}, x_{2}, x_{3}\right) \mapsto\left(-x_{1}, x_{2}, x_{3}\right)$ with respect to the $x_{2} x_{3^{-}}$ plane to preserve the orientation. The quarter-space is quasiconformally equivalent to the half-space $\mathbb{H}$ via mapping $s$ that is a composition of restriction of quasiregular mapping $\left(r, \varphi, x_{2}\right) \mapsto\left(r, 2 \varphi, x_{2}\right)$ and rotation of angle $-\pi / 2$ with respect to the positive $x_{2}$-axis. Above $(r, \varphi)$ refers to polar coordinates of the $x_{3} x_{1}$-plane. By further applying mapping $r \circ I$, the composition of all these mappings gives a quasiconformal mapping $h_{T}: Z^{-} \rightarrow \mathbb{H}$ that maps the end $-\infty$ of $Z$ to $\infty$ and also $h_{T}\left(Z^{-} \cap\left\{x_{3}=0\right\}\right)=\overline{\mathbb{D}} \times\{0\}$ holds. The above steps are illustrated in Figure 2 , The half-lines parallel to the positive $x_{1}$-axes on the right hand side symbolically represent the circles orthogonal to the $x_{2}$ axis going through point $(1,0,0)$.

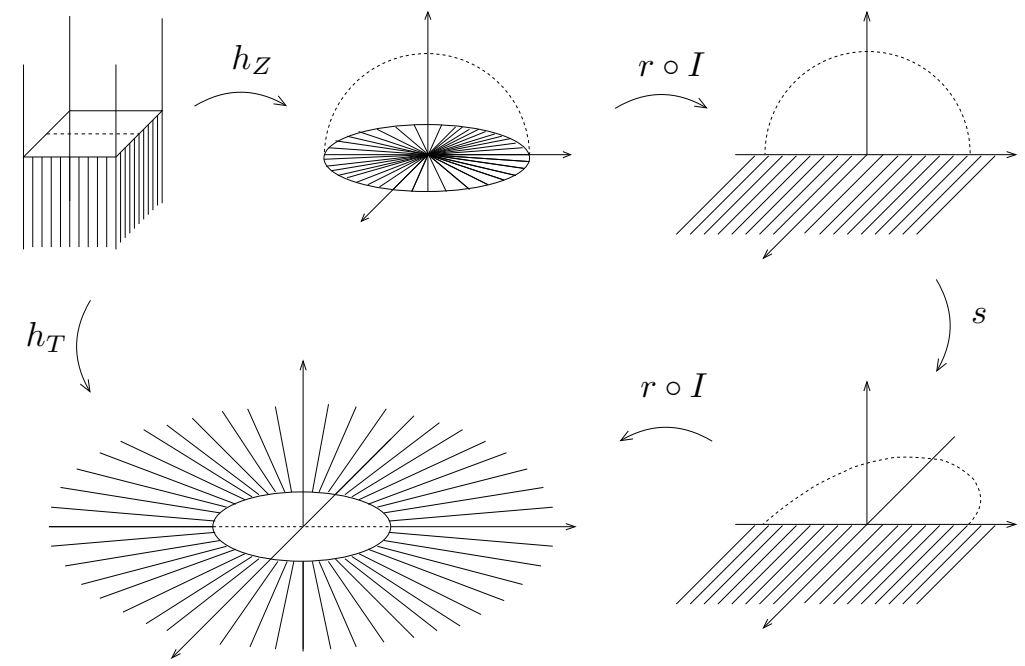

Figure 2. Construction of Tchebychev mapping $h_{T}$ on $\mathbb{S}^{3}$

Let us then extend $h_{T}$ to a quasiregular mapping of $\mathbb{R}^{3}$ onto itself. We use reflections on the faces of $Z^{-}$and $\partial \mathbb{H}$ on the range. This extension, still denoted 
by $h_{T}$, maps half-cylinders alternately onto the upper and lower half-space. By construction, $h_{T}$ is automorphic with respect to the discrete group of isometries $\Gamma$ generated by the translations $x \mapsto x+2 e_{1}$, and $x \mapsto x+2 e_{2}$, and by the two rotations $x \mapsto\left(-x_{1}, x_{2},-x_{3}\right)$ and $x \mapsto\left(x_{1},-x_{2},-x_{3}\right)$.

Now any mapping $A_{\lambda}: x \mapsto \lambda x$, where $\lambda=2,3, \ldots$, induces a $\lambda^{2}$-to- 1 uqr mapping $f_{T}$ of Lattès type on $\mathbb{S}^{3}$ :

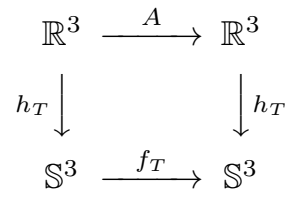

The Julia set of such a mapping is $\mathcal{J}_{f_{T}}=h_{T}\left(\left\{x_{3}=0\right\}\right)=\overline{\mathbb{D}} \times\{0\}$. Its complement, the Fatou set, is the only basin of attraction, and there the iterates $f_{T}^{k}$ converge locally uniformly to the completely invariant fixed point $\infty$. In addition, the infinity point is superattracting, since $f_{T}$ is not locally homeomorphic there [3. The set $\left\{\left(x_{1}, x_{2}, 0\right) \in \mathbb{R}^{3} \mid x_{1}^{2}+x_{2}^{2}>1\right\}$ consisting of the image points of the faces of the infinite cylinders under the mapping $h_{T}$ is only forward invariant. The point $h_{T}(0)$ is a repelling fixed point of the mapping $f_{T}$. The branch set of $f_{T}$ is $h_{T}\left(\left(A_{\lambda}^{-1}\left(B_{h_{T}}\right) \backslash B_{h_{T}}\right)\right.$, where the branch set of the mapping $h_{T}$ constitutes the union of the edges of the cylindrical decomposition of the space and the one skeleton of the unit square lattice in the $x_{1} x_{2}$-plane, and it is denoted by $B_{h_{T}}$. Note that although the branch set of $h_{T}$ is different to that of the Zorich mapping $h_{Z}$ above, the resulting branch set for both induced mappings including local indices are the same: branch lines constitute $2\left(\lambda^{2}-1\right)$ half lines sharing local index 2 and meeting at the origin and infinity with local index $\lambda^{2}$.

3.2. Projective space $\mathbb{P}^{3}$. We construct two uqr mappings of Lattès type acting on the three dimensional projective space $\mathbb{P}^{3}$ : a chaotic branched mapping and a mapping with the Julia set $\mathbb{P}^{2}$. Other uqr mappings (not of Lattès type) on $\mathbb{P}^{3}$ have been constructed with the conformal trap method in [12. The space $\mathbb{P}^{3}$ is, in addition to the sphere, so far the only example of a manifold that supports uqr maps that can be produced both via the trap method and Lattès-type construction.

3.2.1. Chaotic uqr mapping on $\mathbb{P}^{3}$. We recall that a projective space is the space of one-dimensional vector subspaces of a given vector space. The notation $\mathbb{P}^{n}$ denotes the real projective space of dimension $n$ (i.e., the space of one-dimensional vector subspaces of $\left.\mathbb{R}^{(n+1)}\right)$. $\mathbb{P}^{n}$ can also be viewed as the set $\mathbb{S}^{n} /\{x,-x\}$, where $x$ and $-x$ denote the antipodal points.

We wish to present $\mathbb{R}^{3}$ as a branched cover for $\mathbb{P}^{3}$. Therefore we look at the projective space $\mathbb{P}^{3}$ as a block in $\mathbb{R}^{3}$. We subdivide the projective space into two tiles denoted by + and - in Figure 3, The gluing pattern in $\mathbb{R}^{3}$ is described in Figure 4 . Thus in Figure 4 any two adjacent pieces cover the space $\mathbb{P}^{3}$ once. From Figure 4 we can count the Euler characteristic to be $\chi=$ \#vertices $-\#$ edges $+\#$ faces $-\#$ cells $=$ $6-10+6-2=0$, so we are indeed dealing with a manifold.

Now we can define which points on $\mathbb{R}^{3}$ need to be identified to present $\mathbb{R}^{3}$ as a branched cover for $\mathbb{P}^{3}$. Let $e_{1}, e_{2}$ and $e_{3}$ be three orthogonal unit vectors in $\mathbb{R}^{3}$ 


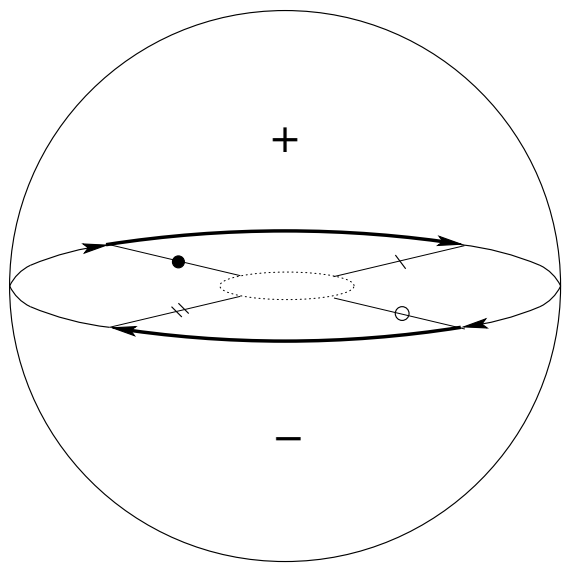

Figure $3 . \mathbb{P}^{3}$

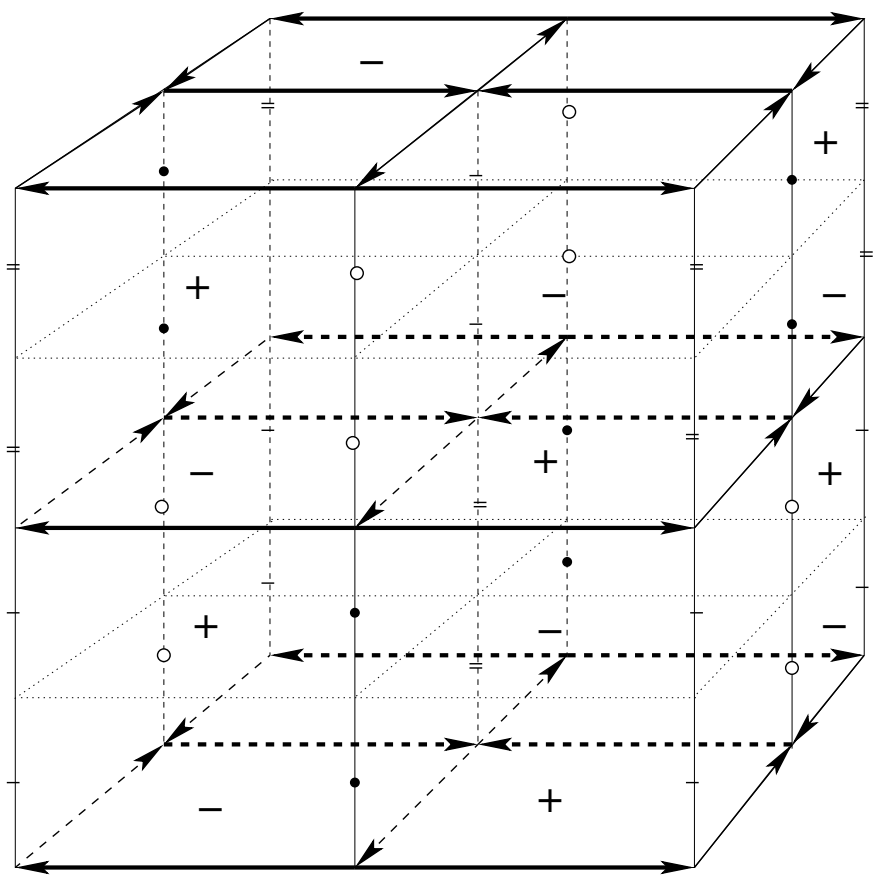

Figure 4 . The gluing pattern of eight copies of $\mathbb{P}^{3}$ 
and let $x_{1} e_{1}+x_{2} e_{2}+x_{3} e_{3}$ be an arbitrary point in $\mathbb{R}^{3}, x_{1}, x_{2}, x_{3} \in \mathbb{R}$. We need to identify all the points in the set

$$
\begin{aligned}
&\left\{\left(x_{1}+2 n_{1}\right) e_{1}+\left(x_{2}+2 m_{1}\right) e_{2}+\left(x_{3}+2 k_{1}\right) e_{3},\right. \\
&\left(2-x_{1}+2 n_{2}\right) e_{1}+\left(2-x_{2}+2 m_{2}\right) e_{2}+\left(x_{3}+2 k_{2}\right) e_{3}, \\
&\left(1-x_{1}+2 n_{3}\right) e_{1}+\left(1-x_{2}+2 m_{3}\right) e_{2}+\left(1+x_{3}+2 k_{3}\right) e_{3}, \\
&\left(2-x_{1}+2 n_{4}\right) e_{1}+\left(x_{2}+2 m_{4}\right) e_{2}+\left(1-x_{3}+2 k_{4}\right) e_{3}, \\
&\left(x_{1}+2 n_{5}\right) e_{1}+\left(2-x_{2}+2 m_{5}\right) e_{2}+\left(1-x_{3}+2 k_{5}\right) e_{3}, \\
&\left(1-x_{1}+2 n_{6}\right) e_{1}+\left(1+x_{2}+2 m_{6}\right) e_{2}+\left(2-x_{3}+2 k_{6}\right) e_{3}, \\
&\left(1+x_{1}+2 n_{7}\right) e_{1}+\left(1-x_{2}+2 m_{7}\right) e_{2}+\left(2-x_{3}+2 k_{7}\right) e_{3}, \\
&\left(1+x_{1}+2 n_{8}\right) e_{1}+\left(1+x_{2}+2 m_{8}\right) e_{2}+\left(1+x_{3}+2 k_{8}\right) e_{3}, \\
&\left.\mid n_{i}, m_{j}, k_{l} \in \mathbb{Z}\right\}
\end{aligned}
$$

in $\mathbb{R}^{3}$ to be the same point on $\mathbb{P}^{3}$, which can be seen from Figure 4 .

Denote the above defined branched covering map by $g: \mathbb{R}^{3} \rightarrow \mathbb{P}^{3}$. This covering map branches on each line of Figure 4. We use the dilation $A_{3}: \mathbb{R}^{3} \rightarrow \mathbb{R}^{3}$, where $A_{3}: x \mapsto 3 x$, to create a uqr mapping. The mappings $A_{3}$ and $g$ induce a mapping $f$ to the manifold $\mathbb{P}^{3}$ :

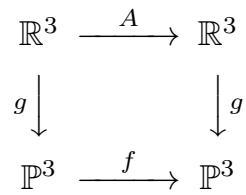

Let us check that the mapping $f$ is a well-defined and uniformly quasiregular mapping of Lattès type. Fix the origin to be at the bottom-left corner of Figure 4 and denote the orthogonal unit vectors again by $e_{1}, e_{2}$ and $e_{3}$. Let $x$ be such a point on $\mathbb{P}^{3}$ that $x_{1} e_{1}+x_{2} e_{2}+x_{3} e_{3}$, where $x_{i} \in[0,2)$ for all $i=1,2,3$, is one of its preimages under $g$. Then the set of preimage points of $x$ on $\mathbb{R}^{3}$ is

$$
\begin{aligned}
g^{-1}(x)=\{ & \left(x_{1}+2 n_{1}\right) e_{1}+\left(x_{2}+2 m_{1}\right) e_{2}+\left(x_{3}+2 k_{1}\right) e_{3}, \\
& \left(2-x_{1}+2 n_{2}\right) e_{1}+\left(2-x_{2}+2 m_{2}\right) e_{2}+\left(x_{3}+2 k_{2}\right) e_{3}, \\
& \left(1-x_{1}+2 n_{3}\right) e_{1}+\left(1-x_{2}+2 m_{3}\right) e_{2}+\left(1+x_{3}+2 k_{3}\right) e_{3}, \\
& \left(2-x_{1}+2 n_{4}\right) e_{1}+\left(x_{2}+2 m_{4}\right) e_{2}+\left(1-x_{3}+2 k_{4}\right) e_{3}, \\
& \left(x_{1}+2 n_{5}\right) e_{1}+\left(2-x_{2}+2 m_{5}\right) e_{2}+\left(1-x_{3}+2 k_{5}\right) e_{3}, \\
& \left(1-x_{1}+2 n_{6}\right) e_{1}+\left(1+x_{2}+2 m_{6}\right) e_{2}+\left(2-x_{3}+2 k_{6}\right) e_{3}, \\
& \left(1+x_{1}+2 n_{7}\right) e_{1}+\left(1-x_{2}+2 m_{7}\right) e_{2}+\left(2-x_{3}+2 k_{7}\right) e_{3}, \\
& \left(1+x_{1}+2 n_{8}\right) e_{1}+\left(1+x_{2}+2 m_{8}\right) e_{2}+\left(1+x_{3}+2 k_{8}\right) e_{3}, \\
& \left.\mid n_{i}, m_{j}, k_{l} \in \mathbb{Z}\right\} .
\end{aligned}
$$


Consequently,

$$
\begin{aligned}
A_{3}\left(g^{-1}(x)\right) \subset\{ & \left(3 x_{1}+2 n_{1}\right) e_{1}+\left(3 x_{2}+2 m_{1}\right) e_{2}+\left(3 x_{3}+2 k_{1}\right) e_{3}, \\
& \left(2-3 x_{1}+2 n_{2}\right) e_{1}+\left(2-3 x_{2}+2 m_{2}\right) e_{2}+\left(3 x_{3}+2 k_{2}\right) e_{3}, \\
& \left(1-3 x_{1}+2 n_{3}\right) e_{1}+\left(1-3 x_{2}+2 m_{3}\right) e_{2}+\left(1+3 x_{3}+2 k_{3}\right) e_{3}, \\
& \left(2-3 x_{1}+2 n_{4}\right) e_{1}+\left(3 x_{2}+2 m_{4}\right) e_{2}+\left(1-3 x_{3}+2 k_{4}\right) e_{3}, \\
& \left(3 x_{1}+2 n_{5}\right) e_{1}+\left(2-3 x_{2}+2 m_{5}\right) e_{2}+\left(1-3 x_{3}+2 k_{5}\right) e_{3}, \\
& \left(1-3 x_{1}+2 n_{6}\right) e_{1}+\left(1+3 x_{2}+2 m_{6}\right) e_{2}+\left(2-3 x_{3}+2 k_{6}\right) e_{3}, \\
& \left(1+3 x_{1}+2 n_{7}\right) e_{1}+\left(1-3 x_{2}+2 m_{7}\right) e_{2}+\left(2-3 x_{3}+2 k_{7}\right) e_{3}, \\
& \left(1+3 x_{1}+2 n_{8}\right) e_{1}+\left(1+3 x_{2}+2 m_{8}\right) e_{2}+\left(1+3 x_{3}+2 k_{8}\right) e_{3} \\
& \left.\mid n_{i}, m_{j}, k_{l} \in \mathbb{Z}\right\}
\end{aligned}
$$

which under the mapping $g$ is again just one point $y:=y_{1} e_{1}+y_{2} e_{2}+y_{3} e_{3}$, where $y_{i} \in[0,2), y_{i}=3 x_{i} \bmod 2$, when $i=1,2,3$. Thus $A_{3}$ descends to a well-defined mapping $f: \mathbb{P}^{3} \rightarrow \mathbb{P}^{3}$.

The discrete group of isometries, $\Gamma$, now consists of all the translations $2 e_{1}, 2 e_{2}$ and $2 e_{3}$, the 180-degree rotation around the vertical middle axis of Figure 4 , translation $e_{1}+e_{2}+2 e_{3}$ and the isometry defined by reflection by $e_{2}$-axis and translation $\frac{1}{2} e_{3}$. Thus the group $\Gamma$ has six generators. The mapping $g$ is automorphic in the strong sense with respect to the group $\Gamma$, and for $A_{3}: x \mapsto 3 x$ it holds that

$$
A_{3} \Gamma A_{3}^{-1} \subset \Gamma \text {. }
$$

Therefore, by Theorem 2.3 the mapping $f: \mathbb{P}^{3} \rightarrow \mathbb{P}^{3}$ is uniformly quasiregular. The degree of this mapping is 27 .

The Julia set $\mathcal{J}_{f}$ is the whole space, which can be seen as follows:

The origin is a repelling fixed point for the mapping $A_{3}: x \mapsto 3 x$. Consider its $\Gamma$-orbit, $\Gamma(0)=\{\gamma(0) \mid \gamma \in \Gamma\}=3 \mathbb{Z}^{3}$. The set $E=\bigcup_{k>0} A_{3}^{-k}(\Gamma(0))$ is a dense subset of $\mathbb{R}^{3}$. Hence, $g(E)$ is a dense subset of $\mathbb{P}^{3}$. We conclude that $\left(f^{k}\right)$ cannot be equicontinuous in a neighbourhood of any point of $\mathbb{P}^{3}$. This means that the Fatou set is empty and the mapping $f$ is chaotic. Point $g(0)$ is a repelling fixed point of the mapping $f$. The branch set of $f$ is the lattice $g\left(\left(A_{3}^{-1} B_{g}\right) \backslash B_{g}\right)$, where $B_{g}$ is the branch set of $g$ that consists of vertical edges and dotted horizontal lines of the subdivision of $\mathbb{R}^{3}$ in Figure 4. This mapping has a generalization that uses $A_{\lambda}$ for arbitrary odd $\lambda>1$.

Remark 3.1. It is interesting to note that essentially the same chaotic mapping is induced if one first takes the cubical subdivision of $\mathbb{R}^{3}$ as in 3.1 .2 and then introduces the antipodal condition by identifying point $\left(x_{1}, x_{2}, x_{3}\right) \in[0,1)^{3}$ with point $\left(1+x_{1}, 1-x_{2}, 1-x_{3}\right)$ in cube $[1,2) \times[0,1)^{2}$. Then one attains three more points due to the identification rules for the sphere: $\left(1-x_{1}, 1+x_{2}, 1-x_{3}\right)$, $\left(1+x_{1}, 1+x_{2}, 1+x_{3}\right)$ and $\left(1-x_{1}, 1-x_{2}, 1+x_{3}\right)$ to correspond to a single point in $\mathbb{P}^{3}$. Each unit cube congruent to $[0,1)^{3}$ then gives a copy of $\mathbb{P}^{3}$ so that the points in neighbouring cubes represent the same points up to reflection with respect to the adjacent face. This identification pattern gives slightly different subdivision of $\mathbb{R}^{3}$ but the induced mappings are the same up to a quasiconformal deformation. It is then possible to use the mapping $A_{\lambda}$, for odd $\lambda>1$ to obtain the induced mapping 
$\tilde{f}: \mathbb{P}^{3} \rightarrow \mathbb{P}^{3}$ via the diagram,

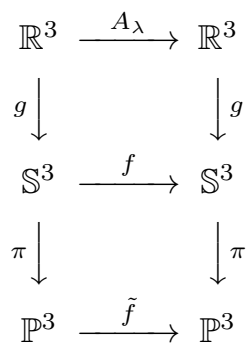

where mapping $\pi: \mathbb{S}^{3} \rightarrow \mathbb{P}^{3}$ is the 2-to-1 covering projection.

Above we saw how the same mapping can be created from the structure of $\mathbb{P}^{3}$ itself. There one can find the corresponding representatives for a sphere from identifications on lines 1, 2, 4 and 5 in (3.5) and for the further subdivision for representatives of the projective space; for example, line 7 in (3.5). The other identifications then follow up to translations modulo 2 from the spherical identifications.

The latter decomposition based on the subdivision of 3.1 .2 is more straightforward to generalize into higher dimensions.

3.2.2. Uqr mapping on $\mathbb{P}^{3}$ with Julia set $\mathbb{P}^{2}$. In 3.1 we constructed the uqr mapping $f_{p w r}: \mathbb{S}^{3} \rightarrow \mathbb{S}^{3}$, a generalization of the power mapping. Now we use this mapping to construct another Lattès-type uqr mapping on $\mathbb{P}^{3}$. If we denote the 2 -to- 1 covering map by $\pi: \mathbb{S}^{3} \rightarrow \mathbb{P}^{3}$, we will get the following commuting diagram for odd $\lambda$ :

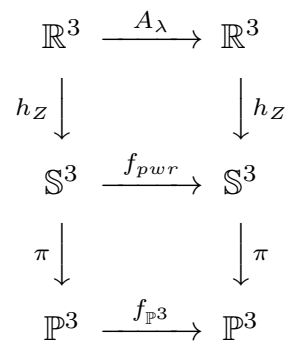

Next, we prove in detail that the mapping $f_{\mathbb{P}^{3}}$ is well defined, and thus by Theorem 2.3 it is a uqr mapping.

Consider $\mathbb{S}^{3}$ as the unit sphere of complex 2-space $\mathbb{C}^{2}$. Let $\tau: \mathbb{S}^{3} \rightarrow \mathbb{S}^{3}$ be the 2-periodic homeomorphism $\tau\left(z_{1}, z_{2}\right)=\left(z_{1} e^{i \pi}, z_{2} e^{i \pi}\right)=\left(-z_{1},-z_{2}\right)$. Now $\mathbb{S}^{3}$ is a universal covering space for $\mathbb{P}^{3}$ and $\tau$ is a generator of the cyclic group of covering translations.

Consider a point $p \in \mathbb{P}^{3}$. It has two preimage points on $\mathbb{S}^{3} \subset \mathbb{C}^{2}$, let us denote them by $\left(\xi_{1}, \xi_{2}, \xi_{3}, t\right)$ and $\left(-\xi_{1},-\xi_{2},-\xi_{3},-t\right)$, where the first and last two coordinates represent a complex number. Thus $\xi_{1}^{2}+\xi_{2}^{2}+\xi_{3}^{2}+t^{2}=1$ and $\xi_{1}, \xi_{2}, \xi_{2}, t \in$ $[-1,1]$.

We can consider the 3 -sphere also as $\mathbb{R}^{3} \cup\{\infty\}$ via the stereographic projection (see Figure 5). Denote the stereographic projection with respect to the north pole by $\sigma: \mathbb{S}^{3} \subset \mathbb{C}^{2} \rightarrow \mathbb{R}^{3} \cup\{\infty\}, \sigma\left(x_{1}, y_{1}, x_{2}, y_{2}\right)=\frac{\left(x_{1}, y_{1}, x_{2}\right)}{1-y_{2}}$. Now we denote the 


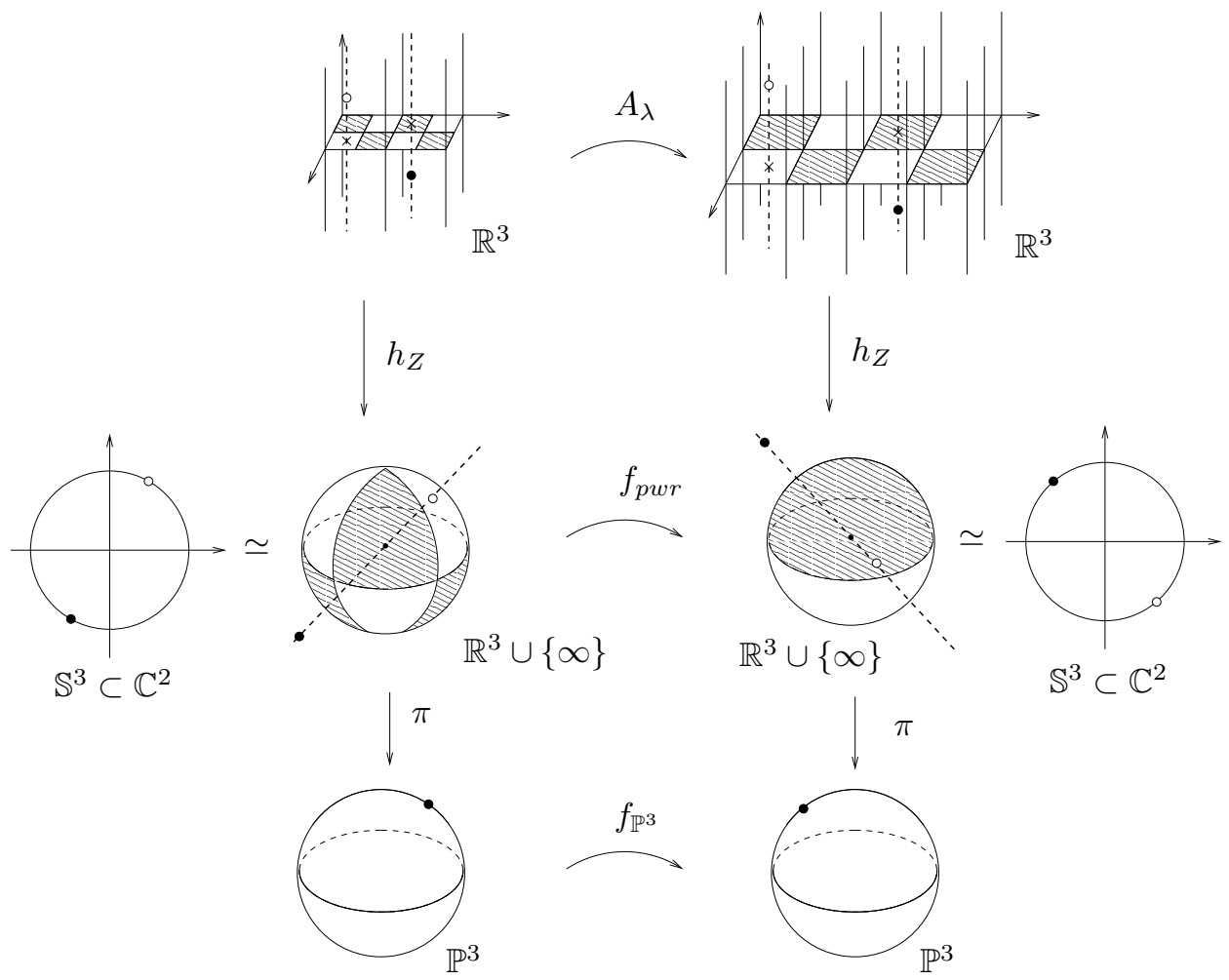

Figure 5. Construction of uqr mapping $f_{\mathbb{P}^{3}}$ on $\mathbb{P}^{3}$

obtained points by

$$
\begin{aligned}
& u_{+}:=\sigma\left(\xi_{1}, \xi_{2}, \xi_{3}, t\right)=\frac{\left(\xi_{1}, \xi_{2}, \xi_{3}\right)}{1-t} \\
& u_{-}:=\sigma\left(-\xi_{1},-\xi_{2},-\xi_{3},-t\right)=\frac{-\left(\xi_{1}, \xi_{2}, \xi_{3}\right)}{1+t} .
\end{aligned}
$$

It then holds that

$$
\left|u_{+}\right|=\sqrt{\frac{1+t}{1-t}}=\frac{1}{\left|u_{-}\right|} .
$$

If we choose $\alpha \in \mathbb{R}$ such that $\left|u_{+}\right|=e^{\alpha}$, then $\left|u_{-}\right|=e^{-\alpha}$ holds.

Denote one preimage point of $u_{+}$in $\mathbb{R}^{3}$ under the Zorich mapping $h_{Z}$ as $(x, y, \alpha)$ for some $x, y \in \mathbb{R}$ (see the definition of $h_{Z}$ in 3.1.1). Hence one preimage point of $u_{-}$is $(1-x, 1+y,-\alpha)$. The whole preimage set of a point $p \in \mathbb{P}^{3}$ in $\mathbb{R}^{3}$ is $h_{Z}^{-1}\left(u_{+}\right) \cup h_{Z}^{-1}\left(u_{-}\right)$, where

$$
\begin{aligned}
h_{Z}^{-1}\left(u_{+}\right)= & \{(x+2 n, y+2 m, \alpha),(2-x+2 k, 2-y+2 l, \alpha) \mid n, m, k, l \in \mathbb{Z}\}, \\
h_{Z}^{-1}\left(u_{-}\right)= & \{(1-x+2 n, 1+y+2 m,-\alpha),(1+x+2 k, 1-y+2 l,-\alpha) \\
& \mid n, m, k, l \in \mathbb{Z}\} .
\end{aligned}
$$


Now we apply dilation $A_{\lambda}(z)=\lambda z$, for $\lambda \in \mathbb{Z}_{+}$and $z \in \mathbb{R}^{3}$. We obtain

$$
\begin{aligned}
A_{\lambda}\left(h_{Z}^{-1}\left(u_{+}\right)\right)= & (\lambda x+2 \lambda n, \lambda y+2 \lambda m, \lambda \alpha), \\
& (2 \lambda-\lambda x+2 \lambda k, 2 \lambda-\lambda y+2 \lambda l, \lambda \alpha) \mid n, m, k, l \in \mathbb{Z}\} .
\end{aligned}
$$

Correspondingly,

$$
\begin{aligned}
A_{\lambda}\left(h_{Z}^{-1}\left(u_{-}\right)\right)=\{ & (\lambda-\lambda x+2 \lambda n, \lambda+\lambda y+2 \lambda m, \lambda \alpha), \\
& (\lambda+\lambda x+2 \lambda k, \lambda-\lambda y+2 \lambda l,-\lambda \alpha) \mid n, m, k, l \in \mathbb{Z}\} .
\end{aligned}
$$

To gain a unique induced mapping $f_{\mathbb{P}^{3}}$, points $A_{\lambda}\left(h_{Z}^{-1}\left(u_{+}\right)\right)$and $A_{\lambda}\left(h_{Z}^{-1}\left(u_{-}\right)\right)$must be mapped onto a single point under $\pi \circ h_{Z}$.

For all $\lambda \in Z_{+}$, points $(2 \lambda-\lambda x+2 \lambda k, 2 \lambda-\lambda y+2 \lambda l, \lambda \alpha)$ can be written in the form $(2-\lambda x+2(\lambda k+\lambda-1), 2-\lambda y+2(\lambda l+\lambda-1), \lambda \alpha)$ hence showing that points $A_{\lambda}\left(h_{Z}^{-1}\left(u_{+}\right)\right)$land to a single point under $\pi \circ h_{Z}$ since

$$
h_{Z} \circ A_{\lambda} \circ h_{Z}^{-1}\left(u_{+}\right)=h_{Z}(\lambda x, \lambda y, \lambda \alpha)
$$

holds.

However, the points $A_{\lambda}\left(h_{Z}^{-1}\left(u_{-}\right)\right)$respect the same symmetry only for odd $\lambda$. Then one can write

$$
\begin{aligned}
& (\lambda-\lambda x+2 \lambda n, \lambda+\lambda y+2 \lambda m,-\lambda \alpha) \\
& =\left(1-\lambda x+2\left(\lambda n+\frac{\lambda-1}{2}\right), 1+\lambda y+2\left(\lambda m+\frac{\lambda-1}{2}\right),-\lambda \alpha\right)
\end{aligned}
$$

and

$$
\begin{aligned}
& (\lambda+\lambda x+2 \lambda k, \lambda-\lambda y+2 \lambda l,-\lambda \alpha) \\
& =\left(1+\lambda x+2\left(\lambda k+\frac{\lambda-1}{2}\right), 1-\lambda y+2\left(\lambda l+\frac{\lambda-1}{2}\right),-\lambda \alpha\right) .
\end{aligned}
$$

Hence, for odd $\lambda$,

$$
h_{Z} \circ A_{\lambda} \circ h_{Z}^{-1}\left(u_{-}\right)=h_{Z}(1-\lambda x, 1+\lambda y,-\lambda \alpha)
$$

holds and gives precisely the antipodal points for those in (3.11). For even $\lambda$ the corresponding points need no more be antipodal and hence the needed symmetry breaks down.

Points (3.11) and (3.12) are of the form $\pm\left(\tilde{\xi}_{1}, \tilde{\xi}_{2}, \tilde{\xi}_{2}, \tilde{t}\right)$ on $\mathbb{S}^{3} \subset \mathbb{C}^{2}$ for some $\tilde{\xi}_{1}, \tilde{\xi}_{2}, \tilde{\xi}_{2}, \tilde{t} \in \mathbb{R}$, giving preimage points of a single point $\tilde{p}$ on $\mathbb{P}^{3}$ under the covering $\operatorname{map} \pi$.

Thus a well-defined mapping $f_{\mathbb{P} 3}$ is induced between the two projective spaces at the bottom of the diagram (3.10) for odd $\lambda>1$. The mapping $f_{\mathbb{P}^{3}}$ is uniformly quasiregular by Theorem 2.3 The Julia set of the mapping $f_{\mathbb{P}^{3}}$ is the set corresponding to the sphere $\mathbb{S}^{2}$ (radius 1 , centered at the origin) in $\mathbb{S}^{3}$. That is, the Julia set is two-dimensional projective space $\mathbb{P}^{2}$ in the three-dimensional projective space $\mathbb{P}^{3}$. The mapping $f_{\mathbb{P}^{3}}$ has only one basin of attraction, since the two basins of attraction in $\mathbb{S}^{3}$ are identified by the covering map $\pi$, and the origin is a superattractive fixed point. The branch set consists of three circles that meet at one point. The degree of the mapping $f_{\mathbb{P}^{3}}$ is $\lambda^{2}$, when dilation $A_{\lambda}$ is used.

Remark 3.2. Note that there is no analogous mapping acting on the projective space that could be induced by the Tchebychev mapping acting on the sphere. This is the case, since the Tchebychev mapping has only one basin of attraction 
and especially only one superattractive fixed point, the infinity. Therefore it is not possible to find the needed antipodal point for the infinity under any subdivision.

3.3. Manifold $\mathbb{S}^{2} \times \mathbb{S}^{1}$. A chaotic Lattès-type uqr mapping acting on $\mathbb{S}^{2} \times \mathbb{S}^{1}$ was constructed by Kangaslampi in [7. We recall this mapping based on a cubical subdivision of the Euclidean space. Furthermore we show that the Zorich mapping can be used to induce the same mapping.

Consider $\mathbb{S}^{2} \times \mathbb{S}^{1}$ as a sphere $\mathbb{S}^{2}$, which has a line segment attached to each of its points and the ends of each line segment identified (see Figure 6)(a)). Now we see that $\mathbb{S}^{2} \times \mathbb{S}^{1}$ has $\mathbb{R}^{3}$ as a branched cover: let us divide $\mathbb{R}^{3}$ into cubes. The base of every other cube is identified with the upper half space of $\mathbb{S}^{2}$, every other with the lower half space (denoted by + and - in Figures 6(a) and 6(b)). The top of the cube is identified with the bottom, thus forming $\mathbb{S}^{1}$ to each point of $\mathbb{S}^{2}$.

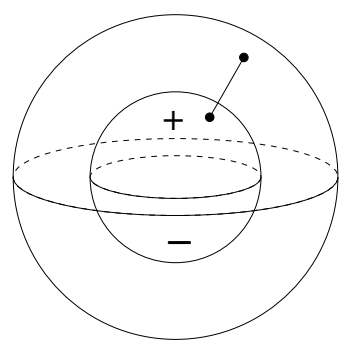

(a) $\mathbb{S}^{2} \times \mathbb{S}^{1}$

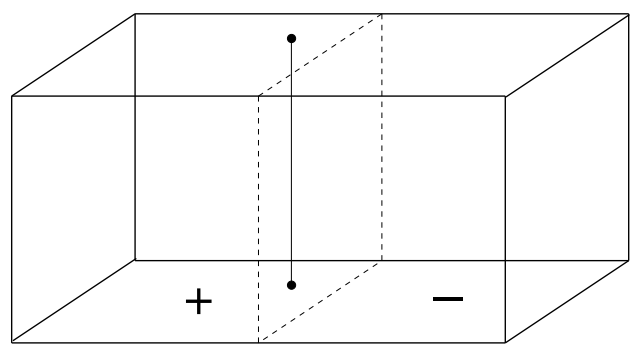

(b) One block in $\mathbb{R}^{3}$ that covers $\mathbb{S}^{2} \times \mathbb{S}^{1}$.

Figure 6 . How to see $\mathbb{S}^{2} \times \mathbb{S}^{1}$ as a block in $\mathbb{R}^{3}$.

To be able to tile $\mathbb{R}^{3}$ with $\mathbb{S}^{2} \times \mathbb{S}^{1}$, we have to make one reproducible block from two images of $\mathbb{S}^{2} \times \mathbb{S}^{1}$ as in Figure 6(b). One building block, which thereby covers $\mathbb{S}^{2} \times \mathbb{S}^{1}$ twice, is presented in Figure 7

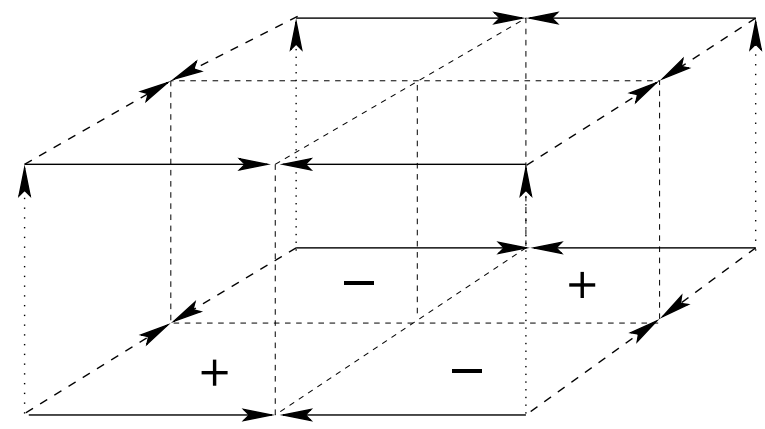

FiguRE 7. One block which covers $\mathbb{S}^{2} \times \mathbb{S}^{1}$ twice.

Let us now define which points in $\mathbb{R}^{3}$ need to be identified to present the whole $\mathbb{R}^{3}$ as a branched cover for $\mathbb{S}^{2} \times \mathbb{S}^{1}$. Let $e_{1}, e_{2}$ and $e_{3}$ be the three orthogonal unit vectors in $\mathbb{R}^{3}$, and let $x_{1}, x_{2}$ and $x_{3} \in \mathbb{R}$ be an arbitrary point in $\mathbb{R}^{3}$. We identify 
all the points of the set

$$
\begin{aligned}
& \left\{\left(x_{1}+n\right) e_{1}+\left(x_{2}+2 m\right) e_{2}+\left(x_{3}+2 k\right) e_{3},\right. \\
& \left(x_{1}+n^{\prime}\right) e_{1}+\left(2-x_{2}+2 m^{\prime}\right) e_{2}+\left(2-x_{3}+2 k^{\prime}\right) e_{3} \\
& \left.\quad \mid n, n^{\prime}, m, m^{\prime}, k, k^{\prime} \in \mathbb{Z}\right\}
\end{aligned}
$$

to be the same point of the manifold. This identification presents $\mathbb{R}^{3}$ as a branched cover for $\mathbb{S}^{2} \times \mathbb{S}^{1}$; see Figure 7 . The branch set is the vertical edge of the skeleton of the tiling.

Define the covering map to be $g: \mathbb{R}^{3} \rightarrow \mathbb{S}^{2} \times \mathbb{S}^{1}$. We use the mappings $A_{2}$ : $\mathbb{R}^{3} \rightarrow \mathbb{R}^{3}$, where $A_{2}: x \mapsto 2 x$ for any $x \in \mathbb{R}^{3}$. The mappings $A_{2}$ and $g$ induce a mapping $f$ to the manifold, and the following diagram commutes:

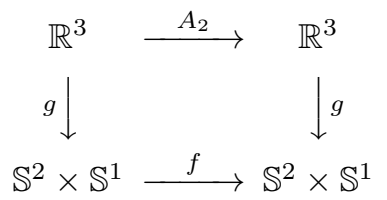

The mapping $f$ is a well-defined and uniformly quasiregular mapping of Lattés type, which we can prove as follows. Let $\left\{e_{1}, e_{2}, e_{3}\right\}$ be an orthonormal basis for $\mathbb{R}^{3}$ and fix the origin to be at the bottom-left corner of Figure 7 . Let $x$ be such a point on $\mathbb{S}^{2} \times \mathbb{S}^{1}$ that $x_{1} e_{1}+x_{2} e_{2}+x_{3} e_{3}$, where $x_{1} \in[0,1)$ and $x_{2}, x_{3} \in[0,2)$, is one of its preimages under $g$. The preimage set of the point $x$ under the covering map $g$ is

$$
\begin{aligned}
g^{-1}(x)=\{ & \left(x_{1}+n\right) e_{1}+\left(x_{2}+2 m\right) e_{2}+\left(x_{3}+2 k\right) e_{3}, \\
& \left(x_{1}+n^{\prime}\right) e_{1}+\left(2-x_{2}+2 m^{\prime}\right) e_{2}+\left(2-x_{3}+2 k^{\prime}\right) e_{3} \\
& \left.\mid n, m, k, n^{\prime}, m^{\prime}, k^{\prime} \in \mathbb{Z}\right\} .
\end{aligned}
$$

Consequently,

$$
\begin{aligned}
A_{2}\left(g^{-1}(x)\right) \subset \quad\{ & \left(2 x_{1}+n\right) e_{1}+\left(2 x_{2}+2 m\right) e_{2}+\left(2 x_{3}+2 k\right) e_{3}, \\
& \left(2 x_{1}+n^{\prime}\right) e_{1}+\left(2-2 x_{2}+2 m^{\prime}\right) e_{2}+\left(2-2 x_{3}+2 k^{\prime}\right) e_{3} \\
& \left.\mid n, m, k, n^{\prime}, m^{\prime}, k^{\prime} \in \mathbb{Z}\right\},
\end{aligned}
$$

which under the mapping $g$ is again just one point $y:=y_{1} e_{1}+y_{2} e_{2}+y_{3} e_{3}$, where $y_{1}$ is the fractional part of $2 x_{1}$, and for $j=2,3, y_{j} \in[0,2), y_{j}=2 x_{j} \bmod 2$. Thus $A_{2}$ descends to a well-defined mapping $f: \mathbb{S}^{2} \times \mathbb{S}^{1} \rightarrow \mathbb{S}^{2} \times \mathbb{S}^{1}$.

In this case, the discrete group of isometries, $\Gamma$, consists of all the translations between the four-parted blocks (see Figure 7) in $\mathbb{R}^{3}$ and the 180-degree rotation around the middle axis of Figure 7 . Thus the group $\Gamma$ has four generators. The mapping $g$ is automorphic in the strong sense with respect to the group $\Gamma$, and for $A_{2}: x \mapsto 2 x$ it holds that $A_{2} \Gamma A_{2}^{-1} \subset \Gamma$. Therefore, by Theorem 2.3, the welldefined mapping $f: \mathbb{S}^{2} \times \mathbb{S}^{1} \rightarrow \mathbb{S}^{2} \times \mathbb{S}^{1}$ is uniformly quasiregular. The degree of the mapping $f$ is 8 . The branch set of $f$ is the six disjoint, unlinked circles defined by the lattice $g\left(\left(A_{2}^{-1} B_{g}\right) \backslash B_{g}\right)$, where $B_{g}$ is the branch set of $g$. The mapping $f$ generalizes to the degree $\lambda^{3}$ mapping when dilation $A_{\lambda}$ is used for arbitrary integer $\lambda>1$.

Remark 3.3. The uqr counterpart of the power mapping constructed in 3.1.1 acts on $\mathbb{R}^{3} \backslash\{0\}$. Space $\mathbb{R}^{3} \backslash\{0\}$ is conformally equivalent to space $S^{2} \times \mathbb{R}$ via mapping 


$$
\begin{gathered}
c: \mathbb{R}^{3} \backslash\{0\} \rightarrow S^{2} \times \mathbb{R},(\theta, \phi, r) \mapsto(\theta, \phi, \log r), \text { since } \\
c^{*} \tilde{g}=\frac{1}{r^{2}} g
\end{gathered}
$$

holds for metric $g=\mathrm{d} r^{2}+r^{2} \mathrm{~d} \theta^{2}+r^{2} \mathrm{~d} \phi^{2}, r>0, \theta \in(0, \pi), \phi \in(0,2 \pi)$ on $\mathbb{R}^{3} \backslash\{0\}$ and metric $\tilde{g}=\mathrm{d} s^{2}+\mathrm{d} \theta^{2}+\mathrm{d} \phi^{2}, s \in \mathbb{R}$ on $\mathbb{S}^{2} \times \mathbb{R}$.

We can further conjugate the power mapping $f_{\text {pwr }}: \mathbb{R}^{3} \backslash\{0\} \rightarrow \mathbb{R}^{3} \backslash\{0\}$ with $c$ to gain a mapping $c \circ f_{p w r} \circ c^{-1}$ acting on $S^{2} \times \mathbb{R}$. By further semiconjugating with the conformal covering mapping $\pi: S^{2} \times \mathbb{R} \rightarrow S^{2} \times S^{1}$, a uqr mapping is attained via equation (2.3):

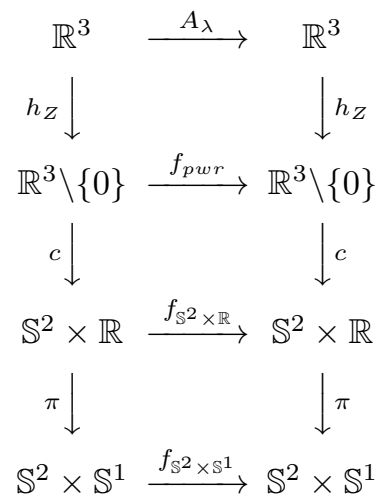

Due to cocompactness the induced mapping is chaotic. The degree of the mapping is $\lambda^{3}$. The branch set consists of $2\left(\lambda^{2}-1\right)$ disjoint, unlinked circles if dilation $A_{\lambda}$ is used.

3.4. Other examples of elliptic 3-manifolds. The only closed elliptic 3-manifolds are those manifolds which are covered by $\mathbb{S}^{3}, \mathbb{S}^{2} \times \mathbb{R}$ or $\mathbb{R}^{3}[6]$. The following theorem for them has been proved by Kangaslampi in 7 :

Theorem 3.4. All quasiregularly elliptic compact oriented 3-dimensional Riemannian manifolds admit a non-trivial uniformly quasiregular mapping.

The case $\mathbb{S}^{3}$ follows from Peltonen's results for spherical space forms in [12]: If $M^{n}$ is a smooth Riemannian manifold with universal covering space $\mathbb{S}^{n}$, then $M^{n}$ admits a branched uniformly quasiregular mapping. This theorem has been proved by using a generalization of the so-called conformal trap method, due to Martin and Iwaniec (see [5]). The Julia sets of these uqr mappings are Cantor sets [5, p. 500]. The uqr mappings constructed with the trap method are not Lattès-type mappings. As we mentioned earlier, the only quotient space of $\mathbb{S}^{3}$ to which a Lattès-type uqr mapping has so far been constructed is the projective 3 -space.

The cases where $M$ is a 3 -dimensional compact Riemannian manifold which has $\mathbb{R}^{3}$ or $\mathbb{S}^{2} \times \mathbb{R}$ as a universal covering has been proved in [7] by direct constructions. There are eight different situations depending on the geometry of the manifold. The constructions follow generally the same idea as the construction on the projective space in Section 3.2.1, and the obtained Lattès-type uqr mappings are chaotic. The manifolds covered by $\mathbb{R}^{3}$ are quotient spaces of the 3 -torus. In Section 4.6 we present a generalized version of the construction method developed in [7. The orientable manifolds covered by $\mathbb{S}^{2} \times \mathbb{R}$ are $\mathbb{S}^{2} \times \mathbb{S}^{1}$ and $\mathbb{P}^{3} \# \mathbb{P}^{3}$. 


\section{LATTÈS-TYPE UQR MAPPINGS IN FOUR DIMENSIONS}

In this chapter we construct some uqr mappings of Lattès type on four-dimensional orientable Riemannian manifolds. Especially we consider their Julia sets, basins of attraction, and branch sets. We see that also in four dimensions the Julia set can be an orientable manifold and its codimension varies from zero to one. The cases that we will consider are collected to Table 1.

TABLE 1. Some Lattès-type uqr mappings and their Julia sets in four dimensions

\begin{tabular}{|c|c|c|}
\hline Manifold & Mapping & Julia set \\
\hline $\mathbb{S}^{4}$ & $f_{\text {cube }}$ & $\mathbb{S}^{4}$ \\
$\mathbb{S}^{4}$ & $f_{\text {pwr }}$ & $\mathbb{S}^{3}$ \\
$\mathbb{S}^{4}$ & $f_{T}$ & $\overline{\mathbb{D}}^{3}$ \\
$\mathbb{S}^{2} \times \mathbb{S}^{2}$ & $f_{\text {cube }}$ & $\mathbb{S}^{2} \times \mathbb{S}^{2}$ \\
$\mathbb{S}^{3} \times \mathbb{S}^{1}$ & $f_{\text {cube }}$ & $\mathbb{S}^{3} \times \mathbb{S}^{1}$ \\
$\mathbb{P}^{3} \times \mathbb{S}^{1}$ & $f_{\text {cube }}$ & $\mathbb{P}^{3} \times \mathbb{S}^{1}$ \\
$\mathbb{P}^{3} \times \mathbb{R}$ & $f_{\text {pwr }}$ & $\mathbb{P}^{3}$ \\
$\mathbb{S}^{2} \times T^{2}$ & $f_{\text {cube }}$ & $\mathbb{S}^{2} \times T^{2}$ \\
$T^{4} / \Gamma$ & $f_{\text {cube }}$ & $T^{4} / \Gamma$ \\
\hline
\end{tabular}

Since there are no orientable spherical space forms other than the sphere itself in even dimensions [17, dimension four also lacks these types of examples.

4.1. Sphere $\mathbb{S}^{4}$. The cubical decomposition in four dimensions gives us a chaotic Lattès-type mapping $f_{\text {cube }}$ on $\mathbb{S}^{4}$. The construction is presented in 3.1 .2 in three dimensions. Here we just take four-dimensional cubes instead and the rest follows analogously as for the three sphere. For the identifications, one subdivides the even integer lattice cubes further into 16 unit cubes which pairwise cover the sphere 8 times. Twice repeated reflections with respect to 3 -faces of unit cubes identify a point $\left(x_{1}, x_{2}, x_{3}, x_{4}\right) \in[0,2) \times[0,1)^{3}$ with 6 different points in $[0,2)^{4}$ whose two coordinates are of the form $2-x_{j}$ and the other two are of the form $x_{k}$. The eighth point $\left(2-x_{1}, 2-x_{2}, 2-x_{3}, 2-x_{4}\right)$ is obtained after 4 repeated reflections.

The Zorich mapping introduced in 3.1.1 can be generalized to higher dimensions as well. We just operate on the first coordinates as we did in the three-dimensional case, and leave the last one to represent the height. That is, we operate on cylinders of the form $\left\{x \in \mathbb{R}^{4} \mid x=y+t e_{4}, y \in Q, t \in \mathbb{R}\right\}$, where $Q$ runs through the set of 3 -cubes into which $\mathbb{R}^{3}$ is subdivided by the planes $x_{k}=i, k=1,2,3, i \in \mathbb{Z}$ [13, p. 15].

Thus the mapping $A: x \mapsto 2 x$ on $\mathbb{R}^{4}$ can be taken to act on $\mathbb{S}^{4}$ by semiconjugating with the Zorich mapping and extending it to the origin and infinity to gain the mapping $h_{Z}: \mathbb{R}^{4} \rightarrow \mathbb{S}^{4}$ :

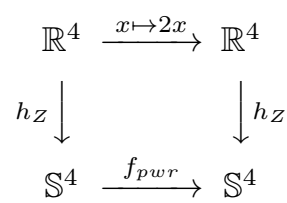


We obtain by Theorem 2.3 a uqr mapping $f_{\text {pwr }}: \mathbb{S}^{4} \rightarrow \mathbb{S}^{4}$. Analogously to the three-dimensional case, the Julia set of this uqr mapping is the 3-dimensional sphere of radius 1 , centered at the origin. The branch set is the two-dimensional set $h_{Z}\left(\left(A_{\lambda}^{-1}\left(B_{h_{Z}}\right)\right) \backslash B_{h_{Z}}\right)$ that is topologically a suspension of the 1-skeleton of a symmetric triangulation of the three-sphere to the origin and infinity. This subdivision of the three-sphere has 16 simplices and 8 vertices. Six vertices can be taken on a two sphere ( 4 along a fixed equator and the 2 poles) and one more both inside and outside the two-sphere. The points of attraction are the origin and the infinity.

The Tchebychev mapping $f_{T}$ defined in 3.1 .3 can be generalized from $\mathbb{S}^{3}$ to other spheres similarly as the power mapping. On $\mathbb{S}^{n}$ let the base of the cylinder in the construction be $(n-1)$-dimensional, and proceed with the remaining coordinate as in the three-dimensional case. The Julia set of $f_{T}$ on $\mathbb{S}^{n}$ is a closed disk of codimension 1 , centered at the origin. Thus, on $\mathbb{S}^{4}$ we have $J_{f_{T}}=\overline{\mathbb{D}}^{3} \times\{0\}$.

4.2. $\mathbb{S}^{2} \times \mathbb{S}^{2}$. Consider both spherical factors of $\mathbb{S}^{2} \times \mathbb{S}^{2}$ subdivided into two unit squares with identifications on the boundary as for the $\mathbb{S}^{2}$-factor in Figure [6). Space $\mathbb{S}^{2} \times \mathbb{S}^{2}$ is then represented as a collection consisting of four unit 4-cubes with induced identifications on the boundary. To produce a branched covering $g: \mathbb{R}^{4} \rightarrow \mathbb{S}^{2} \times \mathbb{S}^{2}$ we subdivide $\mathbb{R}^{4}$ into cubes congruent to $[0,2)^{4}$ with even integer lattice. Each cube is further divided into 16 unit cubes. Let $e_{1}, e_{2}, e_{3}$ and $e_{4}$ denote the four orthogonal unit vectors in $\mathbb{R}^{4}$, and let $x_{1}, x_{2}, x_{3}, x_{4} \in \mathbb{R}$ be an arbitrary point in $\mathbb{R}^{4}$. We identify all the points of the set

$$
\begin{aligned}
& \left\{\left(x_{1}+2 n\right) e_{1}+\left(x_{2}+2 m\right) e_{2}+\left(x_{3}+2 k\right) e_{3}+\left(x_{4}+2 l\right) e_{4},\right. \\
& \left(2-x_{1}+2 n^{\prime}\right) e_{1}+\left(2-x_{2}+2 m^{\prime}\right) e_{2}+\left(x_{3}+2 k^{\prime}\right) e_{3}+\left(x_{4}+2 l^{\prime}\right) e_{4}, \\
& \left(x_{1}+2 n^{\prime \prime}\right) e_{1}+\left(x_{2}+2 m^{\prime \prime}\right) e_{2}+\left(2-x_{3}+2 k^{\prime \prime}\right) e_{3}+\left(2-x_{4}+2 l^{\prime \prime}\right) e_{4}, \\
& \left(2-x_{1}+2 \tilde{n}\right) e_{1}+\left(2-x_{2}+2 \tilde{m}\right) e_{2}+\left(2-x_{3}+2 \tilde{k}\right) e_{3}+\left(2-x_{4}+2 \tilde{l}\right) e_{4} \\
& \left.\quad \mid n, n^{\prime}, n^{\prime \prime}, \tilde{n}, m, m^{\prime}, m^{\prime \prime}, \tilde{m}, k, k^{\prime}, k^{\prime \prime}, \tilde{k}, l, l^{\prime}, l^{\prime \prime}, \tilde{l} \in \mathbb{Z}\right\}
\end{aligned}
$$

to be the same point of the manifold $\mathbb{S}^{2} \times \mathbb{S}^{2}$. This identification induces a branched covering $g: \mathbb{R}^{4} \rightarrow \mathbb{S}^{2} \times \mathbb{S}^{2}$. Each cube congruent to $[0,2)^{4}$ covers space $\mathbb{S}^{2} \times \mathbb{S}^{2}$ four times. The branch set is the two skeleton of the tiling consisting of faces

$$
\left\{(n, m, k, l) \times \mathbb{R}^{2}, \mathbb{R}^{2} \times\left(n^{\prime}, m^{\prime}, k^{\prime}, l^{\prime}\right) \mid n, m, k, l, n^{\prime}, m^{\prime}, k^{\prime}, l^{\prime} \in \mathbb{Z}\right\} .
$$

Denote mapping $A_{\lambda}: \mathbb{R}^{4} \rightarrow \mathbb{R}^{4}$, where $A_{\lambda}: x \mapsto \lambda x$ for any $x \in \mathbb{R}^{4}$ and $\lambda>1$ is a fixed integer. The mappings $A_{\lambda}$ and $g$ induce a mapping $f$ acting on $\mathbb{S}^{2} \times \mathbb{S}^{2}$. We can draw the following diagram:

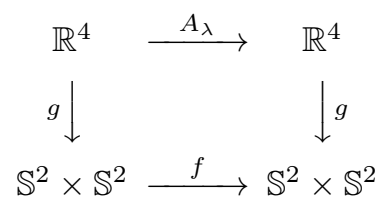

The mapping $f$ is a well-defined and uniformly quasiregular mapping of Lattés type, which we can prove as follows. Let $x$ be a point on $\mathbb{S}^{2} \times \mathbb{S}^{2}$ and $x_{1} e_{1}+x_{2} e_{2}+$ $x_{3} e_{3}+x_{4} e_{4} \in \mathbb{R}^{4}$, where $x_{1}, x_{2}, x_{3}, x_{4} \in[0,2)$, be one of its preimages under $g$. Now we see that the whole preimage set of the point $x$ under the branched covering 
map $g$ is

$$
\begin{aligned}
g^{-1}(x)=\{ & \left(x_{1}+2 n\right) e_{1}+\left(x_{2}+2 m\right) e_{2}+\left(x_{3}+2 k\right) e_{3}+\left(x_{4}+2 l\right) e_{4}, \\
& \left(2-x_{1}+2 n^{\prime}\right) e_{1}+\left(2-x_{2}+2 m^{\prime}\right) e_{2}+\left(x_{3}+2 k^{\prime}\right) e_{3}+\left(x_{4}+2 l^{\prime}\right) e_{4}, \\
& \left(x_{1}+2 n^{\prime \prime}\right) e_{1}+\left(x_{2}+2 m^{\prime \prime}\right) e_{2}+\left(2-x_{3}+2 k^{\prime \prime}\right) e_{3}+\left(2-x_{4}+2 l^{\prime \prime}\right) e_{4}, \\
& \left(2-x_{1}+2 \tilde{n}\right) e_{1}+\left(2-x_{2}+2 \tilde{m}\right) e_{2}+\left(2-x_{3}+2 \tilde{k}\right) e_{3}+\left(2-x_{4}+2 \tilde{l}\right) e_{4} \\
& \left.\mid n, m, k, l, n^{\prime}, m^{\prime}, k^{\prime}, l^{\prime}, n^{\prime \prime}, m^{\prime \prime}, k^{\prime \prime}, l^{\prime \prime}, \tilde{n}, \tilde{m}, \tilde{k}, \tilde{l} \in \mathbb{Z}\right\} .
\end{aligned}
$$

Consequently,

$$
\begin{aligned}
A_{\lambda}\left(g^{-1}(x)\right) & \\
& \subset\left\{\left(\lambda x_{1}+2 n\right) e_{1}+\left(\lambda x_{2}+2 m\right) e_{2}+\left(\lambda x_{3}+2 k\right) e_{3}+\left(\lambda x_{4}+2 l\right) e_{4},\right. \\
& \left(2-\lambda x_{1}+2 n^{\prime}\right) e_{1}+\left(2-\lambda x_{2}+2 m^{\prime}\right) e_{2}+\left(\lambda x_{3}+2 k^{\prime}\right) e_{3}+\left(\lambda x_{4}+2 l^{\prime}\right) e_{4}, \\
& \left(\lambda x_{1}+2 n^{\prime \prime}\right) e_{1}+\left(\lambda x_{2}+2 m^{\prime \prime}\right) e_{2}+\left(2-\lambda x_{3}+2 k^{\prime \prime}\right) e_{3}+\left(2-\lambda x_{4}+2 l^{\prime \prime}\right) e_{4}, \\
& \left(2-\lambda x_{1}+2 \tilde{n}\right) e_{1}+\left(2-\lambda x_{2}+2 \tilde{m}\right) e_{2}+\left(2-\lambda x_{3}+2 \tilde{k}\right) e_{3}+\left(2-\lambda x_{4}+2 \tilde{l}\right) e_{4} \\
& \left.\mid n, m, k, l, n^{\prime}, m^{\prime}, k^{\prime}, l^{\prime}, n^{\prime \prime}, m^{\prime \prime}, k^{\prime \prime}, l^{\prime \prime}, \tilde{n}, \tilde{m}, \tilde{k}, \tilde{l} \in \mathbb{Z}\right\}
\end{aligned}
$$

which under mapping $g$ becomes a single point $y:=y_{1} e_{1}+y_{2} e_{2}+y_{3} e_{3}+y_{4} e_{4}$, where $y_{i} \in[0,2), y_{i}=\lambda x_{i} \bmod 2$ for all $i=1,2,3,4$. Thus $A_{\lambda}$ descends to a well-defined mapping $f: \mathbb{S}^{2} \times \mathbb{S}^{2} \rightarrow \mathbb{S}^{2} \times \mathbb{S}^{2}$.

In this case, the discrete group of isometries, $\Gamma$, consists of the 180-degree rotations $\left(x_{1}, x_{2}, x_{3}, x_{4}\right) \mapsto\left(-x_{1},-x_{2}, x_{3}, x_{4}\right)$ and $\left(x_{1}, x_{2}, x_{3}, x_{4}\right) \mapsto\left(x_{1}, x_{2},-x_{3},-x_{4}\right)$, and all the translations between the blocks $[0,2)^{4}$ in $\mathbb{R}^{4}$. The mapping $g$ is automorphic in the strong sense with respect to the group $\Gamma$, and for $A_{\lambda}: x \mapsto \lambda x$ it holds that $A_{\lambda} \Gamma A_{\lambda}^{-1} \subset \Gamma$. Therefore, by Theorem 2.3. the well-defined mapping $f: \mathbb{S}^{2} \times \mathbb{S}^{2} \rightarrow \mathbb{S}^{2} \times \mathbb{S}^{2}$ is uniformly quasiregular. The degree of the mapping $f$ is $\lambda^{4}$. The branch set of $f$ is the lattice $g\left(\left(A_{\lambda}^{-1} B_{g}\right) \backslash B_{g}\right)$ of form $\left\{\mathbb{S}^{2} \times\left\{q_{i}\right\} \cup\left\{p_{j}\right\} \times \mathbb{S}^{2} \mid i, j \in\right.$ $\left.\left\{1,2, \ldots, 2\left(\lambda^{2}-1\right)\right\}\right\}$. Due to cocompactness the mapping $g$ is chaotic.

4.3. $\mathbb{S}^{3} \times \mathbb{S}^{1}$. Consider the first spherical factor of $\mathbb{S}^{3} \times \mathbb{S}^{1}$ subdivided into two unit cubes with boundary identifications induced by reflections with respect to adjacent 2 -face. The second factor is considered as a unit interval with identified endpoints. Space $\mathbb{S}^{3} \times \mathbb{S}^{1}$ is then represented as a collection consisting of two unit 4-cubes with induced identifications on the boundary. To produce a branched covering $g: \mathbb{R}^{4} \rightarrow \mathbb{S}^{3} \times \mathbb{S}^{1}$ we subdivide $\mathbb{R}^{4}$ into cubes congruent to $[0,2)^{3} \times[0,1)$. Each such cube is further divided into eight unit cubes. Let $e_{1}, e_{2}, e_{3}$ and $e_{4}$ denote the four orthogonal unit vectors in $\mathbb{R}^{4}$, and let $x_{1}, x_{2}, x_{3}, x_{4} \in \mathbb{R}$ be coordinates of a preimage point of an arbitrary point $x \in \mathbb{S}^{3} \times \mathbb{S}^{1}$ under $g$. We identify all the points of the set

$$
\begin{aligned}
& \left\{\left(x_{1}+2 n_{1}\right) e_{1}+\left(x_{2}+2 n_{2}\right) e_{2}+\left(x_{3}+2 n_{3}\right) e_{3}+\left(x_{4}+n_{4}\right) e_{4},\right. \\
& \left(2-x_{1}+2 m_{1}\right) e_{1}+\left(2-x_{2}+2 m_{2}\right) e_{2}+\left(x_{3}+2 m_{3}\right) e_{3}+\left(x_{4}+m_{4}\right) e_{4}, \\
& \left(x_{1}+2 k_{1}\right) e_{1}+\left(2-x_{2}+2 k_{2}\right) e_{2}+\left(2-x_{3}+2 k_{3}\right) e_{3}+\left(x_{4}+k_{4}\right) e_{4}, \\
& \left(2-x_{1}+2 l_{1}\right) e_{1}+\left(x_{2}+2 l_{2}\right) e_{2}+\left(2-x_{3}+2 l_{3}\right) e_{3}+\left(x_{4}+l_{4}\right) e_{4} \\
& \left.\quad \mid n_{i}, m_{i}, k_{i}, l_{i} \in \mathbb{Z}, i=1,2,3,4\right\}
\end{aligned}
$$

to be the same point of the manifold $\mathbb{S}^{3} \times \mathbb{S}^{1}$. This identification induces a branched covering $g: \mathbb{R}^{4} \rightarrow \mathbb{S}^{3} \times \mathbb{S}^{1}$. Each cube congruent to $[0,2)^{3} \times[0,1)$ covers space $\mathbb{S}^{3} \times \mathbb{S}^{1}$ 
four times. The two-dimensional branch set is the product of the 1-skeleton of the cubical subdivision of $\mathbb{R}^{3}$ and $\mathbb{R}$.

Analogously to the case $\mathbb{S}^{2} \times \mathbb{S}^{1}$ one can use the mapping $A_{\lambda}: \mathbb{R}^{4} \rightarrow \mathbb{R}^{4}$, where $A_{\lambda}: x \mapsto \lambda x$ for any $x \in \mathbb{R}^{4}$ and $\lambda>1$ is a fixed integer. The mappings $A_{\lambda}$ and $g$ induce a mapping $f$ acting on $\mathbb{S}^{3} \times \mathbb{S}^{1}$ and the following diagram commutes:

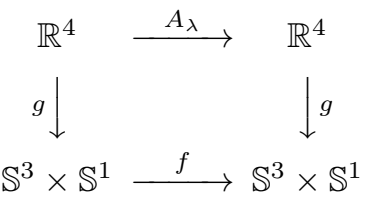

The mapping $f$ is a well-defined and uniformly quasiregular mapping of Lattés type. For the proof, one can write down the sets $g^{-1}(x)$ and $A_{\lambda}\left(g^{-1}(x)\right)$ for an arbitrary point $x \in \mathbb{S}^{3} \times \mathbb{S}^{1}$. The fact that $g\left(A_{\lambda}\left(g^{-1}(x)\right)\right)$ is a single point $y \in \mathbb{S}^{3} \times \mathbb{S}^{1}$ for arbitrary $\lambda>1$ follows from identical calculations for the factors as in case $\mathbb{S}^{2} \times \mathbb{S}^{1}$ in 3.3

In this case, the discrete group of isometries, $\Gamma$, consists of the 180-degree rotations $\left(x_{1}, x_{2}, x_{3}, x_{4}\right) \mapsto\left(-x_{1},-x_{2}, x_{3}, x_{4}\right),\left(x_{1}, x_{2}, x_{3}, x_{4}\right) \mapsto\left(x_{1},-x_{2},-x_{3}, x_{4}\right)$, and $\left(x_{1}, x_{2}, x_{3}, x_{4}\right) \mapsto\left(-x_{1}, x_{2},-x_{3}, x_{4}\right)$, and all the translations between the blocks $[0,2)^{3} \times[0,1)$ in $\mathbb{R}^{4}$. Thus the group $\Gamma$ has seven generators. The mapping $g$ is automorphic in the strong sense with respect to the group $\Gamma$, and for $A_{\lambda}: x \mapsto \lambda x$ it holds that $A_{\lambda} \Gamma A_{\lambda}^{-1} \subset \Gamma$. Therefore, by Theorem 2.3. the well-defined mapping $f: \mathbb{S}^{3} \times \mathbb{S}^{1} \rightarrow \mathbb{S}^{3} \times \mathbb{S}^{1}$ is uniformly quasiregular. The degree of the mapping $f$ is $\lambda^{4}$. The two-dimensional branch set of $f$ is the lattice $g\left(\left(A_{\lambda}^{-1} B_{g}\right) \backslash B_{g}\right)$ which is a product of $\mathbb{S}^{1}$ with a subset of the 1 -skeleton consisting of edges of a symmetric subdivision of the three-sphere into 16 cubes. The edges of a cube containing eight cubes are excluded. Due to cocompactness, the mapping $g$ is chaotic.

Remark 4.1. Via the uqr counterpart of the power mapping acting on $\mathbb{R}^{4} \backslash\{0\}$ and conformal equivalence $c: \mathbb{R}^{4} \backslash\{0\} \rightarrow \mathbb{S}^{3} \times \mathbb{R},\left(\varphi_{1}, \varphi_{2}, \varphi_{3}, r\right) \mapsto\left(\varphi_{1}, \varphi_{2}, \varphi_{3}, \log r\right)$ the same mapping as above is induced to act on $\mathbb{S}^{3} \times \mathbb{S}^{1}$ :

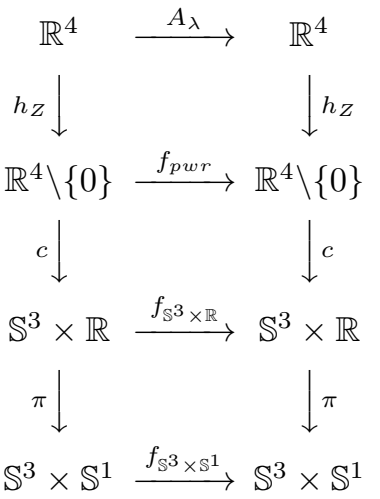

4.4. $\mathbb{P}^{3} \times \mathbb{S}^{1}$. Starting from the subdivision of $\mathbb{R}^{4}$ as for the chaotic mapping for the space $\mathbb{S}^{3} \times \mathbb{S}^{1}$ we introduce the antipodal condition for the first factor and cyclic identification for the second factor by identifying point $\left(x_{1}, x_{2}, x_{3}, x_{4}\right) \in[0,1)^{4}$ with point $\left(1+x_{1}, 1-x_{2}, 1-x_{3}, x_{4}\right)$ in cube $[1,2) \times[0,1)^{3}$. Then one attains three more points due to the identification rules for the sphere: $\left(1-x_{1}, 1+x_{2}, 1-x_{3}, x_{4}\right)$, $\left(1+x_{1}, 1+x_{2}, 1+x_{3}, x_{4}\right)$ and $\left(1-x_{1}, 1-x_{2}, 1+x_{3}, x_{4}\right)$ to correspond to a single 
point in $\mathbb{P}^{3} \times \mathbb{S}^{1}$. Each unit cube congruent to $[0,1)^{4}$ then gives a copy of $\mathbb{P}^{3} \times \mathbb{S}^{1}$ under induced branched covering $\tilde{g}=\pi \circ g: \mathbb{R}^{4} \rightarrow \mathbb{P}^{3} \times \mathbb{S}^{1}$. If $A_{\lambda}$ is dilation $x \mapsto \lambda x$ for odd integer $\lambda>1$, then the induced mapping $\tilde{f}: \mathbb{P}^{3} \times \mathbb{S}^{1} \rightarrow \mathbb{P}^{3} \times \mathbb{S}^{1}$ is obtained via the diagram:

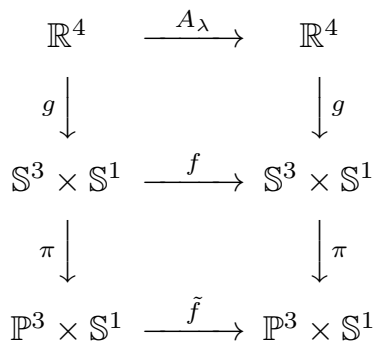

The induced mapping acting on $\mathbb{S}^{3} \times \mathbb{S}^{1}$ is uniquely defined for the arbitrary integer $\lambda>1$, but the identification of points $\left(x_{1}, x_{2}, x_{3}, x_{4}\right) \in[0,1)^{4}$ with point $\left(1+x_{1}, 1-x_{2}, 1-x_{3}, x_{4}\right)$ in cube $[1,2) \times[0,1)^{3}$ is coherent only for odd $\lambda$. This follows since then

$$
\begin{aligned}
& A_{\lambda}\left(\left(1+x_{1}+2 n\right) e_{1}+\left(1-x_{2}+2 m\right) e_{2}+\left(1-x_{3}+2 k\right) e_{3}+\left(x_{4}+l\right) e_{4}\right) \\
& \quad=\left(\lambda+\lambda x_{1}+2 \lambda n\right) e_{1}+\left(\lambda-\lambda x_{2}+2 \lambda m\right) e_{2}+\left(\lambda-\lambda x_{3}+2 \lambda k\right) e_{3}+\left(\lambda x_{4}+\lambda l\right) e_{4} \\
& \quad \subset\left(1+\lambda x_{1}+2 n\right) e_{1}+\left(1-\lambda x_{2}+2 m\right) e_{2}+\left(1-\lambda x_{3}+2 k\right) e_{3}+\left(\lambda x_{4}+l\right) e_{4}
\end{aligned}
$$

holds for $n, m, k, l \in \mathbb{Z}$.

Remark 4.2. Via the uqr counterpart of the power mapping acting on $\mathbb{R}^{4} \backslash\{0\}$ and the 2-to-1 covering $\pi: \mathbb{S}^{3} \rightarrow \mathbb{P}^{3}$ for the first factor of $\mathbb{S}^{3} \times \mathbb{R}$, we get an induced mapping acting on $\mathbb{P}^{3} \times \mathbb{R}$ :

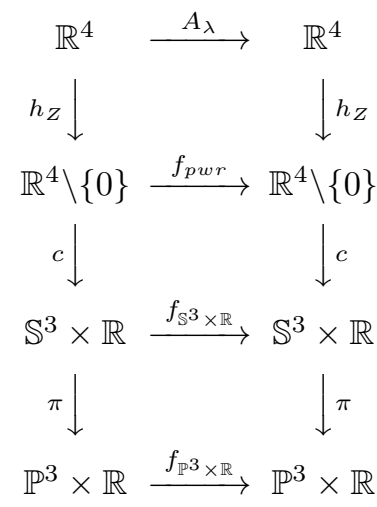

The achieved mapping acting on noncompact $\mathbb{P}^{3} \times \mathbb{R}$ is interesting, since it has the projective 3 -space as the Julia set. This property is not inherited for the quotient map acting on $\mathbb{P}^{3} \times \mathbb{S}^{1}$ which agrees with the above chaotic mapping.

4.5. $\mathbb{S}^{2} \times T^{2}$. Consider the representatives of manifold $\mathbb{S}^{2} \times T^{2}$ in $\mathbb{R}^{4}$ to consist of the block $[0,2) \times[0,1)^{3}$ with boundary identifications for $\mathbb{S}^{2} \times \mathbb{S}^{1}$ factor as in case 3.3. For the last factor, 0 and 1 are identified. A block $[0,2)^{2} \times[0,1)^{2}$ is translated via $\left(x_{1}, x_{2}, x_{3}, x_{4}\right) \rightarrow\left(x_{1}+2 m, x_{2}+2 n, x_{3}+k, x_{4}+l\right), n, m, k, l \in \mathbb{Z}$ to tile the Euclidean 4-space. The block $[0,2)^{2} \times[0,1)^{2}$ (and each translate) contains two representatives of manifold $\mathbb{S}^{2} \times T^{2}$ after identification of a point $\left(x_{1}, x_{2}, x_{3}, x_{4}\right) \in[0,2) \times[0,1)^{3}$ and $\left(2-x_{1}, 2-x_{2}, x_{3}, x_{4}\right) \in[0,2) \times[1,2] \times[0,1)^{2}$ respectively. These identifications 
induce a branched covering $g: \mathbb{R}^{4} \rightarrow \mathbb{S}^{2} \times T^{2}$ whose branch set is $\mathbb{Z}^{2} \times \mathbb{R}^{2}$. The given lattice of $\mathbb{R}^{4}$ is invariant under dilation $A_{\lambda}: x \mapsto \lambda x$ and descends into a mapping $f_{\lambda}: \mathbb{S}^{2} \times T^{2} \rightarrow \mathbb{S}^{2} \times T^{2}$ for arbitrary integer $\lambda>1$ :

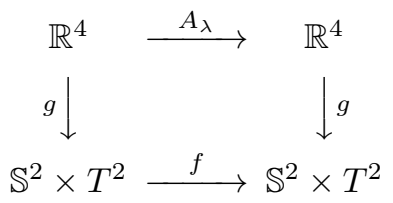

The degree of the mapping $f_{\lambda}$ is $\lambda^{4}$. The branch set of $f$ is $2\left(\lambda^{2}-1\right)$ disjoint, unlinked 2-tori defined by the lattice $g\left(\left(A_{\lambda}^{-1} B_{g}\right) \backslash B_{g}\right)$, where $B_{g}$ is the branch set of $g$.

4.6. $T^{4}$ and its quotient spaces. Perhaps the most basic Lattès-type uqr mapping comes from dilation in $\mathbb{R}^{4}$ and the usual covering map $\pi: \mathbb{R}^{4} \rightarrow T^{4}$ (each 1 -cube in $\mathbb{R}^{4}$ covers the torus once):

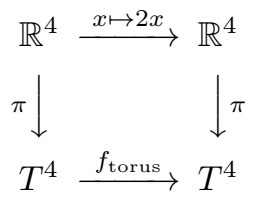

Thus we get a chaotic uqr map $f_{\text {torus }}$ on $T^{4}$.

Let us consider the quotient spaces $T^{4} / \Gamma$ of the torus $T^{4}$. The group $\Gamma$ consists of the translation subgroup and the rotation subgroup. By the characterization by Rinow [15. Theorem 13, p. 373] there exist coordinates $\left\{e_{i}\right\}_{i=1}^{4}$ of the covering space $\mathbb{R}^{4}$ with the following properties:

(1) The elements of the translation part are of the form $x \mapsto x+\sum_{i=1}^{4} m_{i} e_{i}$, $m_{i} \in \mathbb{Z}$.

(2) The general element is of the form $x \mapsto x+\sum_{i=1}^{4} \frac{n_{i}}{g} e_{i}, n_{i} \in \mathbb{Z}$, where $g$ is the order of the rotational part.

(3) The matrix of $R$ representing the rotational part, when presented in this fixed base, has only integer entries.

We present a general method of constructing Lattès-type uqr mappings on manifolds $T^{4} / \Gamma$.

Theorem 4.3. A Lattès-type uqr map $f_{q}: T^{4} / \Gamma \rightarrow T^{4} / \Gamma$ arises as a solution of the equation

$$
\left(f_{q} \circ \pi\right)(x)=(\pi \circ A)(x),
$$

where the dilation $A: \mathbb{R}^{4} \rightarrow \mathbb{R}^{4}$ is

$$
A: x \mapsto(\lambda g+1) x,
$$

$g$ is the order of the rotation matrix $R, \lambda$ is an integer, and $\pi$ is a conformal covering projection.

Proof. We have to show that $A$ preserves the needed lattice identifications, i.e. that the mapping $f_{q}$ is well defined. Then by Theorem 2.3, the solution $f_{q}$ to the Schröder functional equation is a uqr mapping. 
Let $x$ be an arbitrary point in $T^{4} / \Gamma$. Then its preimage points under $\pi$ on $\mathbb{R}^{4}$ are of the form

$$
R(x)+\sum_{i=1}^{4} \frac{n_{i}}{g} e_{i}
$$

where the coefficients $n_{i}$ are integers and an $R$ matrix with integer entries. If we now multiply this preimage point by $\lambda g+1$, we get a point of the form

$$
R((\lambda g+1) x)+(\lambda g+1) \sum_{i=1}^{4} \frac{n_{i}}{g} e_{i}=R((\lambda g+1) x)+\sum_{i=1}^{4} \frac{n_{i}}{g} e_{i}+\lambda \sum_{i=1}^{4} n_{i} e_{i} .
$$

The last sum just takes the point to another representative of $T^{4} / \Gamma$ on $\mathbb{R}^{4}$. Therefore, by the covering map $\pi$, each point of this form is mapped to $(\lambda g+1) x$. Thus the mapping

$$
f_{q}=\pi \circ A \circ \pi^{-1}
$$

is well defined on $T^{4} / \Gamma$, and by Theorem $2.3, f_{q}$ is uniformly quasiregular.

Remark 4.4. The same method works as well in higher dimensions.

\section{LATTÈS-TYPE UQR MAPPINGS IN FIVE DIMENSIONS}

The situation in dimension five is described in Table 2 The power mapping, the Tchebychev mapping and the chaotic mapping from the cubical decomposition acting on the 5 sphere give the basic examples. The projective space $\mathbb{P}^{5}$ admits both a chaotic uqr map and a map with nonorientable Julia set $\mathbb{P}^{4}$. All possible product spaces including spherical, torus or projective space components are chaotic. The same method as in dimension four provides us with chaotic uqr mappings on the quotient spaces of the five-torus (Remark 4.4).

TABLE 2. Julia sets of Lattès-type uqr mappings in five dimensions

\begin{tabular}{|c|c|c|}
\hline Manifold & Mapping & Julia set \\
\hline $\mathbb{S}^{5}$ & $f_{\text {cube }}$ & $\mathbb{S}^{5}$ \\
$\mathbb{S}^{5}$ & $f_{\text {pwr }}$ & $\mathbb{S}^{4}$ \\
$\mathbb{S}^{5}$ & $f_{T}$ & $\bar{D}^{4}$ \\
$\mathbb{P}^{5}$ & $f_{\text {cube }}$ & $\mathbb{P}^{5}$ \\
$\mathbb{P}^{5}$ & $f_{\text {pwr }}$ & $\mathbb{P}^{4}$ \\
$\mathbb{S}^{4} \times \mathbb{S}^{1}$ & $f_{\text {cube }}$ & $\mathbb{S}^{4} \times \mathbb{S}^{1}$ \\
$\mathbb{S}^{3} \times \mathbb{S}^{2}$ & $f_{\text {cube }}$ & $\mathbb{S}^{3} \times \mathbb{S}^{2}$ \\
$\mathbb{S}^{2} \times \mathbb{S}^{2} \times \mathbb{S}^{1}$ & $f_{\text {cube }}$ & $\mathbb{S}^{2} \times \mathbb{S}^{2} \times \mathbb{S}^{1}$ \\
$\mathbb{S}^{3} \times T^{2}$ & $f_{\text {cube }}$ & $\mathbb{S}^{3} \times T^{2}$ \\
$\mathbb{S}^{2} \times T^{3}$ & $f_{\text {cube }}$ & $\mathbb{S}^{2} \times T^{3}$ \\
$T^{5}$ & $f_{\text {cube }}$ & $T^{5}$ \\
$\mathbb{P}^{3} \times \mathbb{S}^{2}$ & $f_{\text {cube }}$ & $\mathbb{P}^{3} \times \mathbb{S}^{2}$ \\
\hline
\end{tabular}


5.1. Chaotic mappings in dimension five. The cubical decomposition for the five-dimensional sphere that gives a chaotic Lattès-type mapping $f_{\text {cube }}$ acting on $\mathbb{S}^{5}$ is attained as follows. Each cube in the even integer lattice is further subdivided into 32 unit cubes which pairwise cover the sphere 16 times. Twice repeated reflections with respect to 4 -faces of unit cubes identify a point $\left(x_{1}, x_{2}, x_{3}, x_{4}, x_{5}\right) \in[0,1)^{5}$ with 10 different points in $[0,2)^{5}$ whose two coordinates are of the form $2-x_{j}$ and the other three are of the form $x_{k}$. The same point is also identified with five more points in $[0,2)^{5}$ that are obtained after 4 repeated reflections. Those points have four coordinates of form $2-x_{j}$ and one of form $x_{k}$.

To obtain the chaotic mapping for the projective space $\mathbb{P}^{5}$, the antipodal condition can be given by identifying point $\left(x_{1}, x_{2}, x_{3}, x_{4}, x_{5}\right) \in[0,1)^{5}$ with point $\left(1+x_{1}\right.$, $\left.1-x_{2}, 1-x_{3}, 1-x_{4}, 1-x_{5}\right) \in[1,2) \times[0,1)^{4}$. Then one attains 15 more points due to the identification rules for the sphere: four more with one factor of the form $1+x_{k}$ and others of the form $1-x_{j}$, ten with three factors of the form $1+x_{k}$ and the other two of the form $1-x_{j}$; one with all factors of the form $1+x_{k}$. This gives, altogether, 32 copies of projective space in block $[0,2)^{5}$ and each of its translates. It is then possible to use the mapping $A_{\lambda}$, for odd $\lambda>1$ to obtain the induced mapping $\tilde{f}: \mathbb{P}^{5} \rightarrow \mathbb{P}^{5}$ via diagram

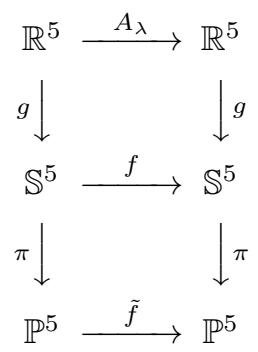

where mapping $\pi: \mathbb{S}^{5} \rightarrow \mathbb{P}^{5}$ is the 2-to-1 covering projection. The degree of the mapping $f$ and $\tilde{f}$ is $\lambda^{5}$. The mapping $f$ acting on the sphere works also for even $\lambda$.

For spaces $\mathbb{S}^{4} \times \mathbb{S}^{1}, \mathbb{S}^{3} \times \mathbb{S}^{2}, \mathbb{S}^{3} \times T^{2}, \mathbb{S}^{2} \times \mathbb{S}^{2} \times \mathbb{S}^{1}, \mathbb{S}^{2} \times T^{3}$ and $T^{5}$ one gets a mapping acting on it according to the diagram

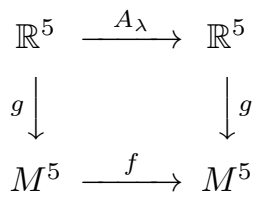

where $M^{5}$ is any of the above spaces and $\lambda>1$ is any integer. All the induced mappings $f$ are of degree $\lambda^{5}$. In all cases the starting point is a cubical even integer lattice subdivision of $\mathbb{R}^{5}$. In the presence of an $\mathbb{S}^{1}$-factor, one takes a unit interval cyclic identification pattern to the corresponding direction. For an $\mathbb{S}^{2}$ factor, one takes two coordinates $x_{k}$ and $x_{k+1}, k \in\{1,2,3,4\}$ and identifies points having the corresponding coordinates $2-x_{k}$ and $2-x_{k+1}$ modulo translations by two units to the directions of chosen axes. For an $\mathbb{S}^{3}$ factor, we take three coordinates $x_{k}$, $x_{k+1}, x_{k+2}, k \in\{1,2,3\}$ and identify four different points in each even integer lattice block having the corresponding coordinates of the forms $2-x_{j}$ and $2-x_{l}$, $j, l \in\{k, k+1, k+2\}, j \neq l$ modulo translations by two units to the directions of chosen axes. 
In case of $\mathbb{S}^{4} \times \mathbb{S}^{1}$, a block congruent to $[0,2)^{4} \times[0,1)$ contains 16 unit cubes that pairwise give a copy of the space. Hence block $[0,2)^{4} \times[0,1)$ contains eight copies of manifold $\mathbb{S}^{4} \times \mathbb{S}^{1}$. For $\mathbb{S}^{3} \times \mathbb{S}^{2}$ a block congruent to $[0,2)^{5}$ contains 32 unit cubes and according to the above rules, four of them is needed to give one copy of the space. Cube $[0,2)^{5}$ hence covers manifold $\mathbb{S}^{3} \times \mathbb{S}^{2}$ eight times. For $\mathbb{S}^{2} \times \mathbb{S}^{2} \times \mathbb{S}^{1}$ a block congruent to $[0,2)^{4} \times[0,1)$ contains 16 unit cubes and according to the above rules, four of them is needed to give one copy of the space. Hence, block $[0,2)^{4} \times[0,1)$ contains four copies of manifold $\mathbb{S}^{2} \times \mathbb{S}^{2} \times \mathbb{S}^{1}$. For space $\mathbb{S}^{3} \times T^{2}$ a block congruent to $[0,2)^{3} \times[0,1)^{2}$ contains 8 unit cubes that pairwise give a copy of the space. Hence block $[0,2)^{3} \times[0,1)^{2}$ contains four copies of manifold $\mathbb{S}^{3} \times T^{2}$. For space $\mathbb{S}^{2} \times \mathbb{S}^{2} \times \mathbb{S}^{1}$ a block congruent to $[0,2)^{2} \times[0,1)^{3}$ contains 4 unit cubes that pairwise give a copy of the space. Hence block $[0,2)^{2} \times[0,1)^{3}$ contains two copies of manifold $\mathbb{S}^{2} \times \mathbb{S}^{2} \times \mathbb{S}^{1}$.

5.2. Codimension one Julia sets. Analogous to the three-dimensional case one gets both the Zorich mapping and the Tchebychev counterpart acting on $\mathbb{S}^{5}$. For $\mathbb{P}^{5}$, a mapping with non-orientable space $\mathbb{P}^{4}$ as a Julia set is induced.

For the 5 -sphere, a Zorich block is set $[0,2)^{4} \times \mathbb{R}$ and all its translates $x_{i} \mapsto$ $x_{i}+2 n, n \in \mathbb{Z}$ with respect to the first four coordinates. Each block covers $\mathbb{S}^{5}$ (or rather $\mathbb{R}^{5} \backslash\{0\}$ ) eight times according to the identification rules for $\mathbb{S}^{4}$. There is no identification for the last factor. The degree of the induced mapping acting on $\mathbb{S}^{5}$ is $\lambda^{4}$ for any dilation $x \mapsto \lambda x$ with integer $\lambda>1$.

The induced mapping for the projective space is given by the following diagram:

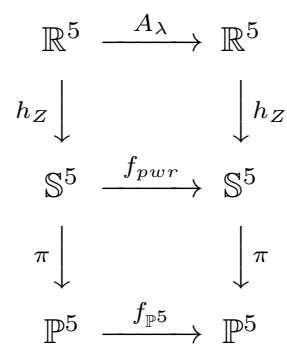

Analogous to the three-dimensional case, the covering translations are induced by mapping $\left(z_{1}, z_{2}, z_{3}\right) \mapsto\left(-z_{1},-z_{2},-z_{3}\right)$. Stereographic projection $\sigma: \mathbb{S}^{5} \subset \mathbb{C}^{3} \rightarrow$ $\mathbb{R}^{5} \cup\{\infty\}$ then gives two related points

$$
\begin{aligned}
& u_{+}:=\sigma\left(\xi_{1}, \xi_{2}, \xi_{3}, \xi_{4}, \xi_{5}, t\right)=\frac{\left(\xi_{1}, \xi_{2}, \xi_{3}, \xi_{4}, \xi_{5}\right)}{1-t} \\
& u_{-}:=\sigma\left(-\xi_{1},-\xi_{2},-\xi_{3},-\xi_{4},-\xi_{5},-t\right)=\frac{-\left(\xi_{1}, \xi_{2}, \xi_{3}, \xi_{4}, \xi_{5}\right)}{1+t},
\end{aligned}
$$

on the projective space. As in three dimensions, the relation

$$
\left|u_{+}\right|=\sqrt{\frac{1+t}{1-t}}=\frac{1}{\left|u_{-}\right|}
$$

holds and we can choose $\alpha \in \mathbb{R}$ such that $\left|u_{+}\right|=e^{\alpha}$ and $\left|u_{-}\right|=e^{-\alpha}$ holds. The set $h_{Z}^{-1}\left(u_{+}\right)$contains eight points with the last coordinate $\alpha$ in each block $[0,2)^{4} \times \mathbb{R}$ and its translate according to the identification rules for $\mathbb{S}^{4}$ for the first four coordinates. Similarly set $h_{Z}^{-1}\left(u_{-}\right)$contains eight points with last coordinate $-\alpha$ in each block $[0,2)^{4} \times \mathbb{R}$. There the identification pattern is given by identifying 
point $\left(x_{1}, x_{2}, x_{3}, x_{4},-\alpha\right) \in[0,1)^{4} \times \mathbb{R}$ and $\left(1+x_{1}, 1-x_{2}, 1-x_{3}, 1-x_{4},-\alpha\right) \in$ $[1,2) \times[0,1)^{3} \times \mathbb{R}$. The remaining six points in the same block are defined by the identification rule for the sphere $\mathbb{S}^{4}$ with respect to the first four coordinates. All the coordinates for $A_{\lambda}\left(h_{Z}^{-1}\left(u_{+}\right)\right), A_{\lambda}\left(h_{Z}^{-1}\left(u_{-}\right)\right)$are of the same form as in the three-dimensional case. Hence, any odd $\lambda>1$ will also do in this case.

\section{General Results in $n$ Dimensions}

6.1. Chaotic mappings. All manifolds of the form

$$
\tilde{M}=\mathbb{S}^{n_{1}} \times \cdots \times \mathbb{S}^{n_{i}} \times T^{m_{1}} \times \cdots \times T^{m_{j}} \times \mathbb{P}^{l_{1}} \times \cdots \times \mathbb{P}^{l_{k}}
$$

support nontrivial chaotic uqr maps. Here we interpret $T^{1}=S^{1}$, integers $n_{q}>1$, $m_{r} \geq 1$ and odd $l_{p}>1, q=1, \ldots, i, r=1, \ldots, j, p=1, \ldots, k$. Denote further $N=\sum_{q=1}^{i} n_{q}, M=\sum_{r=1}^{j} m_{r}, L=\sum_{p=1}^{k} l_{p}$. We assume $M+N+L \geq 3$ but of course the spherical/torus/projective component need not be present at all. In that case, we define $M=0$ or $N=0$ or $L=0$ correspondingly.

The starting point is a cubical decomposition of $\mathbb{R}^{M+N+L}$. For coordinates corresponding to spherical and projective factors, we fix an even integer lattice and integer lattice related to torus coordinates. Each block $[0,2)^{N+L} \times[0,1)^{M}$ and its translate contains $2^{N-1+L}$ copies of the space. The identifications inside each block are given by the even number of reflections with respect to integer lattice faces. For an even dimensional sphere $S^{2 n}$, this gives all together

$$
\sum_{k=1}^{n}\left(\begin{array}{c}
2 n \\
2 k
\end{array}\right)
$$

copies of $S^{2 n}$. For odd dimensional sphere factor $S^{2 n+1}$ one gets correspondingly

$$
\sum_{k=1}^{n}\left(\begin{array}{c}
2 n+1 \\
2 k
\end{array}\right)
$$

copies of $S^{2 n+1}$ under the even number of reflections with respect to codimension one faces. Each spherical component $S^{n_{q}}$ has $2^{n_{q}}$ unit $n_{q}$-cubes for the corresponding coordinates inside each block that pairwise give $2^{n_{q}-1}$ copies related to this factor.

If projective spaces $\mathbb{P}^{l_{k}}$ are present, then one can proceed as for the same dimensional spherical factor $\mathbb{S}^{l_{k}}$ and introduce the antipodal condition between point $\left(x_{1}, \ldots, x_{N+M+1}, \ldots, x_{N+M+L}\right)$ in $[0,2)^{N} \times[0,1)^{M+L}$ and point $\left(x_{1}, \ldots, 1+\right.$ $\left.x_{N+M+1}, 1-x_{N+M+2}, \ldots, 1-x_{N+M+L}\right)$ in $[0,2)^{N} \times[0,1)^{M} \times[1,2) \times[0,1)^{L-1}$. The rest of the $2^{l_{k}}$ identification follow from the identifications rules for the spherical factor $\mathbb{S}^{l_{k}}$. To obtain a well-defined mapping in the case where projective space factors exist, one needs to assume that the dilation $A_{\lambda}: \mathbb{R}^{M+N+L} \rightarrow \mathbb{R}^{M+N+L}$, $x \mapsto \lambda x$ is given for odd integer $\lambda>1$ :

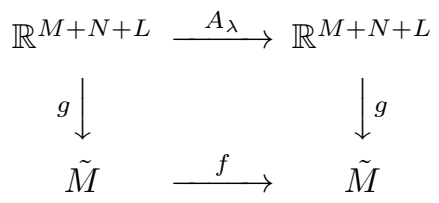

The degree of the mapping $f$ is $\lambda^{M+N+L}$. 
6.2. Codimension one Julia sets. Analogously to the low dimensional cases, one gets both the Zorich mapping and the Tchebychev counterpart acting on any $\mathbb{S}^{n}$. For the projective space $\mathbb{P}^{n}$, a mapping with non-orientable space $\mathbb{P}^{n-1}$ as a Julia set is induced for odd $n$.

For the $n$-sphere, a Zorich block is set $[0,2)^{n-1} \times \mathbb{R}$ and all its translates $x_{i} \mapsto$ $x_{i}+2 m, m \in \mathbb{Z}$ with respect to the first $n-1$ coordinates. Each block covers $\mathbb{S}^{n}$ (or rather $\mathbb{R}^{n} \backslash\{0\}$ ) $2^{n-2}$ times according to the identification rules for $\mathbb{S}^{n-1}$. There is no identification for the last factor. The degree of the induced mapping acting on $\mathbb{S}^{n}$ is $\lambda^{n-1}$ for any dilation $x \mapsto \lambda x$ with integer $\lambda>1$.

The induced mapping for the projective space is given by the following diagram:

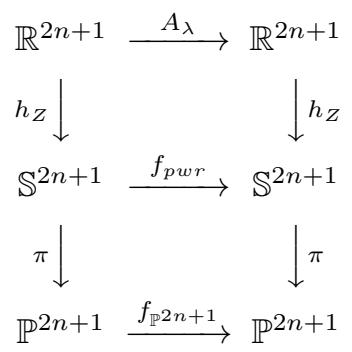

Analogous to the three and five-dimensional cases, the covering translations are induced by mapping $\left(z_{1}, \ldots, z_{2 n-1}\right) \mapsto\left(-z_{1}, \ldots,-z_{2 n-1}\right)$. Stereographic projection $\sigma: \mathbb{S}^{2 n+1} \subset \mathbb{C}^{n+1} \rightarrow \mathbb{R}^{2 n+1} \cup\{\infty\}$ then gives two related points

$$
\begin{aligned}
& u_{+}:=\sigma\left(\xi_{1}, \ldots, \xi_{2 n+1}, t\right)=\frac{\left(\xi_{1}, \ldots, \xi_{2 n+1}\right)}{1-t} \\
& u_{-}:=\sigma\left(-\xi_{1}, \ldots,-\xi_{2 n+1},-t\right)=\frac{-\left(\xi_{1}, \ldots, \xi_{2 n+1}\right)}{1+t}
\end{aligned}
$$

on the projective space. As in the lower dimensions, the relation

$$
\left|u_{+}\right|=\sqrt{\frac{1+t}{1-t}}=\frac{1}{\left|u_{-}\right|}
$$

holds and we can choose $\alpha \in \mathbb{R}$ such that $\left|u_{+}\right|=e^{\alpha}$ and $\left|u_{-}\right|=e^{-\alpha}$ holds. The set $h_{Z}^{-1}\left(u_{+}\right)$contains $2^{2 n-1}$ points with the last coordinate $\alpha$ in each block $[0,2)^{2 n} \times \mathbb{R}$ and its translate according to the identification rules for $\mathbb{S}^{2 n}$ for the first four coordinates. Similarly, set $h_{Z}^{-1}\left(u_{-}\right)$contains $2^{2 n-1}$ points with the last coordinate $-\alpha$ in each block $[0,2)^{2 n} \times \mathbb{R}$. There the identification pattern is given by identifying point $\left(x_{1}, \ldots, x_{2 n},-\alpha\right) \in[0,1)^{2 n} \times \mathbb{R}$ and $\left(1+x_{1}, 1-x_{2}, \ldots, 1-\right.$ $\left.x_{2 n},-\alpha\right) \in[1,2) \times[0,1)^{2 n-1} \times \mathbb{R}$. The rest $2^{2 n-1}-2$ points in the same block are defined by the identification rule for the sphere $\mathbb{S}^{2 n}$ with respect to the first $2 n$ coordinates. All the coordinates for $A_{\lambda}\left(h_{Z}^{-1}\left(u_{+}\right)\right), A_{\lambda}\left(h_{Z}^{-1}\left(u_{-}\right)\right)$are of the same form as in the low dimensional cases. Hence, any odd $\lambda>1$ will also do in this case.

\section{ACKNOWLEDGMENT}

We are deeply grateful to Gaven Martin for his continuous enthusiasm, encouragement, and generosity in sharing mathematics. 


\section{REFERENCES}

1. Zoltán Balogh, Fässler Katrin, and Kirsi Peltonen, Uniformly quasiregular mappings on the compactified Heisenberg group, J. Geom. Anal. (2010) DOI 10.1007/S12220-010-9205-5.

2. Alan F. Beardon, Iteration of rational functions, Graduate Texts in Mathematics, vol. 132, Springer-Verlag, New York, 1991, Complex analytic dynamical systems. MR.1128089 $(92 \mathrm{j}: 30026)$

3. Aimo Hinkkanen, Gaven J. Martin, and Volker Mayer, Local dynamics of uniformly quasiregular mappings, Math. Scand. 95 (2004), no. 1, 80-100. MR.2091483 (2005f:37094)

4. Tadeusz Iwaniec and Gaven Martin, Quasiregular semigroups, Ann. Acad. Sci. Fenn. Math. 21 (1996), no. 2, 241-254. MR1404085 (97i:30032)

5. - Geometric function theory and non-linear analysis, Oxford Mathematical Monographs, The Clarendon Press Oxford University Press, New York, 2001. MR1859913 (2003c:30001)

6. Jorma Jormakka, The existence of quasiregular mappings from $\mathbf{R}^{3}$ to closed orientable 3manifolds, Ann. Acad. Sci. Fenn. Ser. A I Math. Dissertationes (1988), no. 69, 44. MR973719 (90b:57005)

7. Riikka Kangaslampi, Uniformly quasiregular mappings on elliptic Riemannian manifolds, Ann. Acad. Sci. Fenn. Ser. A I Math. Dissertationes (2008), no. 151, 69.

8. S. Lattès, Sur l'itération des substitutions rationnelles et les fonctions de Poincarè, C. R. Acad. Sci. Paris 166 (1918), 26-28.

9. Gaven J. Martin, Branch sets of uniformly quasiregular maps, Conform. Geom. Dyn. 1 (1997), 24-27 (electronic). MR.1454921 (98d:30032)

10. Volker Mayer, Uniformly quasiregular mappings of Lattès type, Conform. Geom. Dyn. 1 (1997), 104-111 (electronic). MR.1482944 (98j:30017)

11. - Quasiregular analogues of critically finite rational functions with parabolic orbifold, J. Anal. Math. 75 (1998), 105-119. MR1655826 (2000a:30043)

12. Kirsi Peltonen, Examples of uniformly quasiregular mappings, Conform. Geom. Dyn. 3 (1999), 158-163 (electronic). MR.1718708 (2001i:30017)

13. Seppo Rickman, Quasiregular mappings, Ergebnisse der Mathematik und ihrer Grenzgebiete (3) [Results in Mathematics and Related Areas (3)], vol. 26, Springer-Verlag, Berlin, 1993. MR:1238941 (95g:30026)

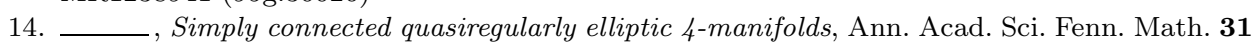
(2006), no. 1, 97-110. MR2210111 (2006k:30041)

15. Willi Rinow, Die innere Geometrie der metrischen Räume, Die Grundlehren der mathematischen Wissenschaften, Bd. 105, Springer-Verlag, Berlin, 1961. MR0123969 (23:A1290)

16. Pekka Tukia, On two-dimensional quasiconformal groups, Ann. Acad. Sci. Fenn. Ser. A I Math. 5 (1980), no. 1, 73-78. MR595178 (82c:30031)

17. Joseph A. Wolf, Spaces of constant curvature, McGraw-Hill Book Co., New York, 1967. MR0217740 (36:829)

Department of Mathematics and Computer Science, Technische Universiteit Eindhoven, P.O. Box 513, 5600 MB Eindhoven, The Netherlands

E-mail address: 1.j.astola@tue.nl

Department of Mathematics and Systems Analysis, Aalto University, P.O. Box 11100, 00076 Aalto, Finland

E-mail address: riikka.kangaslampi@tkk.fi

Department of Mathematics and Systems Analysis, Aalto University, P.O. Box 11100, 00076 Aalto, Finland

E-mail address: kirsi.peltonen@helsinki.fi 